\title{
Active-sterile neutrino oscillations at INO-ICAL over a wide mass-squared range
}

Tarak Thakore, ${ }^{a, b}$ Moon Moon Devi, ${ }^{c}$ Sanjib Kumar Agarwalla ${ }^{d, e, f}$ and Amol Dighe ${ }^{g}$

${ }^{a}$ Louisiana State University, Baton Rouge, Louisiana 70803, U.S.A.

${ }^{b}$ Instituto de Fisica Corpuscular, CSIC - Universitat de València, c/Catedràtico Josè Beltràn 2, E-46980 Paterna, Valencia, Spain

${ }^{c}$ Department of Physics, Tezpur University, Assam 784028, India

${ }^{d}$ Institute of Physics, Sachivalaya Marg, Sainik School Post, Bhubaneswar 751005, India

${ }^{e}$ Homi Bhabha National Institute, Anushakti Nagar, Mumbai 400085, India

${ }^{f}$ International Centre for Theoretical Physics, Strada Costiera 11, 34151 Trieste, Italy

${ }^{g}$ Tata Institute of Fundamental Research, Homi Bhabha Road, Colaba, Mumbai 400005, India

E-mail: tarak.thakore@ific.uv.es, devimm@tezu.ernet.in, sanjib@iopb.res.in, amol@tifr.res.in

ABSTRACT: We perform a detailed analysis for the prospects of detecting active-sterile oscillations involving a light sterile neutrino, over a large $\Delta m_{41}^{2}$ range of $10^{-5} \mathrm{eV}^{2}$ to $10^{2} \mathrm{eV}^{2}$, using 10 years of atmospheric neutrino data expected from the proposed $50 \mathrm{kt}$ magnetized ICAL detector at the INO. This detector can observe the atmospheric $\nu_{\mu}$ and $\bar{\nu}_{\mu}$ separately over a wide range of energies and baselines, making it sensitive to the magnitude and sign of $\Delta m_{41}^{2}$ over a large range. If there is no light sterile neutrino, ICAL can place competitive upper limit on $\left|U_{\mu 4}\right|^{2} \lesssim 0.02$ at $90 \%$ C.L. for $\Delta m_{41}^{2}$ in the range $(0.5-5) \times 10^{-3} \mathrm{eV}^{2}$. For the same $\left|\Delta m_{41}^{2}\right|$ range, ICAL would be able to determine its sign, exploiting the Earth's matter effect in $\mu^{-}$and $\mu^{+}$events separately if there is indeed a light sterile neutrino in Nature. This would help identify the neutrino mass ordering in the four-neutrino mixing scenario.

Keywords: Neutrino Physics, Beyond Standard Model

ArXiv EPrint: 1804.09613 


\section{Contents}

1 Introduction and motivation 1

2 Neutrino mixing and oscillations in four flavors 4

2.1 Mass ordering schemes and configurations 4

2.2 The lepton mixing matrix 5

2.3 Earth matter effects $\quad 7$

2.4 Oscillograms in $E_{\nu}-\cos \theta_{\nu}$ plane 8

3 Event spectra at ICAL and identifying crucial $\left(E_{\mu}, \cos \theta_{\mu}\right)$ bins $\quad 9$

3.1 Event distributions in energy and zenith angle 9

$\begin{array}{ll}3.2 \text { Useful regions in the } E_{\mu}-\cos \theta_{\mu} \text { plane } & 12\end{array}$

4 Constraining active-sterile mixing $\quad 14$

4.1 Exclusion contours in the $\Delta m_{41}^{2}-\left|U_{\mu 4}\right|^{2}$ plane $\quad 15$

4.1.1 Dependence on $\left|U_{e 4}\right|$ and the mass ordering scheme 16

$\begin{array}{ll}\text { 4.1.2 Contribution of rate, energy and direction measurements } & 17\end{array}$

$\begin{array}{lll}4.2 & \text { Comparison with other experiments } & 18\end{array}$

5 Exploring features of $\Delta \boldsymbol{m}_{41}^{2} \quad \mathbf{2 0}$

5.1 Precision in the determination of $\Delta m_{41}^{2} \quad 20$

$\begin{array}{ll}5.2 & \text { Determination of the sign of } \Delta m_{41}^{2}\end{array} 22$

$6 \quad$ Summary and concluding remarks $\quad 24$

\section{Introduction and motivation}

Unraveling neutrino properties has become an ongoing enterprise in the intensity frontier of the high energy particle physics, both experimentally and theoretically [1-3]. Active attempts are being made to probe their masses, mixings, interactions, Dirac vs. Majorana nature, and so on [4]. Over the last two decades, several word-class experiments involving solar [5-14], atmospheric [15, 16], reactor [17-22], and accelerator [23-29] neutrinos have firmly established neutrino flavor oscillations, an engrossing example of a quantum mechanical phenomenon working at the macroscopic scale.

Most of the data from the above mentioned experiments fit well into the standard three-flavor oscillation picture of neutrinos [30-32]. The three-neutrino $(3 \nu)$ oscillation scheme is characterized by six fundamental parameters: (i) three leptonic mixing angles $\left(\theta_{12}, \theta_{13}, \theta_{23}\right)$, (ii) one Dirac CP phase $\left(\delta_{\mathrm{CP}}\right)$, and (iii) two independent mass-squared 
differences ${ }^{1}\left(\Delta m_{21}^{2}\right.$ and $\left.\Delta m_{32}^{2}\right)$. However, not all the oscillation data are compatible with this three-flavor oscillation picture [33]. There are anomalous experiments (for recent reviews see $[34,35])$, which point towards oscillations with substantially large mass-squared difference $\left(\Delta m^{2} \sim 1 \mathrm{eV}^{2}\right)$ as compared to the well-known solar and atmospheric mass splittings. For example, the LSND experiment observed the appearance of $\bar{\nu}_{e}$ events in a $\bar{\nu}_{\mu}$ beam, which can be explained in a two-flavor oscillation framework with $\Delta m^{2} \sim 1 \mathrm{eV}^{2}[36$, 37]. Another indication came from the short-baseline (SBL) Gallium radioactive source experiments GALLEX [6] and SAGE [7], where the disappearance of $\nu_{e}$ was observed. Several SBL reactor antineutrino experiments noticed a deficit in the observed $\bar{\nu}_{e}$ event rates [38] in comparison with that expected from the calculation of reactor antineutrino fluxes $[39,40]$. This so-called reactor neutrino anomaly also strengthened the case in favor of neutrino oscillations driven by $\Delta m^{2} \sim 1 \mathrm{eV}^{2}$. Recently, new model-independent hints in favor of SBL $\bar{\nu}_{e}$ oscillations have emerged from the reactor experiments NEOS [41] and DANSS [42]. By performing the combined analysis of the spectral ratios of these two experiments, the authors of ref. [43] have obtained an indication $(\sim 3.7 \sigma)$ in favor of SBL $\bar{\nu}_{e}$ oscillations $^{2}$ with $\Delta m^{2} \approx 1.3 \mathrm{eV}^{2}$. A recent reanalysis of the ILL reactor data [54] also claims a $\sim 3 \sigma$ evidence for a light sterile neutrino with $\Delta m^{2} \sim 1 \mathrm{eV}^{2}$.

All these anomalies recorded at the SBL experiments cannot be explained with the help of three sub-eV massive neutrinos $\nu_{1}, \nu_{2}, \nu_{3}$ and require the existence of a fourth mass eigenstate $\nu_{4}$ at the $\mathrm{eV}$ scale. This cannot couple to $W$ and $Z$ bosons due to the LEP bounds [55] on the number of the weakly interacting light neutrino state. Hence it has to be a gauge singlet, and can reveal its presence only through its mixing with the active neutrinos. This non-interacting neutrino is popularly known as the "sterile" neutrino. New more sensitive SBL experiments have been planned (see the reviews in refs. [5659]) to test the existence of sterile neutrino by observing the typical $L / E$ pattern in the oscillations driven by the new mass-squared splitting $\Delta m_{41}^{2} \sim 1 \mathrm{eV}^{2}$. Apart from the SBL oscillation experiments, the light sterile neutrino can also have visible effects in ongoing [6062] or upcoming [63-67] long-baseline experiments. Signals of a sterile neutrino may be observed at the existing or planned multi-purpose water- or ice-based large detectors like Super-Kamiokande [49], IceCube [50, 68-71], or DeepCore [72, 73], or during the neutrino burst from a galactic supernova $[74,75]$. The $\beta$-decay and neutrinoless double $\beta$-decay experiments can also feel the presence of a light sterile neutrino (see the review [57] and the references therein). The possible existence of a light sterile neutrino can also have profound implications in cosmology [56, 76, 77].

The sterile neutrino is an elementary particle beyond the Standard Model (SM) and the possible discovery of a light sterile neutrino would prove that there is new physics beyond

\footnotetext{
${ }^{1}$ We define $\Delta m_{i j}^{2} \equiv m_{i}^{2}-m_{j}^{2}$, where $m_{i}$ 's are the masses of the neutrino mass eigenstates $\nu_{i}$ 's, arranged in the decreasing order of electron flavour content. To address the solar neutrino anomaly, we need $\Delta m_{21}^{2} \approx$ $7.5 \times 10^{-5} \mathrm{eV}^{2}$ and to resolve the atmospheric neutrino anomaly, we require $\left|\Delta m_{32}^{2}\right| \approx 2.5 \times 10^{-3} \mathrm{eV}^{2}$.

${ }^{2}$ However, there are SBL experiments like CCFR [44], CDHSW [45], and SciBooNE-MiniBooNE [46, 47] that have observed null results while searching for muon flavor disappearance associated with this high oscillation frequency. The same is also true for the long-baseline experiment MINOS [48] and the atmospheric neutrino experiments Super-Kamiokande [49] and IceCube [50]. Thus, there are tensions among various data sets, while trying to fit all of them in a four-neutrino framework [34, 35, 51-53]. Therefore, the existence of a light sterile neutrino is still inconclusive.
} 
the SM at low-energies, which is completely orthogonal to new physics searches at highenergies at the Large Hadron Collider (LHC). There are several interesting motivations to search for a light sterile neutrino at mass scales different than the eV scale. For instance, the possible existence of a super-light sterile neutrino [78-82], which weakly mixes with the active neutrinos and has a mass very close to the active ones $\left(\Delta m_{41}^{2} \approx 10^{-5} \mathrm{eV}^{2}\right)$, may explain the suppression of the upturn in the energy spectrum of solar neutrino events below $8 \mathrm{MeV}[11,12,14]$. A very light sterile neutrino at a mass scale smaller than $0.1 \mathrm{eV}$ could affect the oscillations of reactor antineutrinos [83-87]. A light sterile neutrino having a mass of a few eV can help in nucleosynthesis of heavy elements inside the supernova [88-90]. A relatively heavy sterile neutrino with mass around $\mathrm{keV}$ scale can act as warm dark matter in the context of the Neutrino Minimal Standard Model ( $\nu$ MSM) [91-94]. Therefore, it makes perfect sense to investigate the presence of a light sterile neutrino over a wide range of mass scale. Recently, the Daya Bay Collaboration also has performed such a search and has obtained bounds on the active-sterile mixing parameters in the mass range of $2 \times 10^{-4} \mathrm{eV}^{2} \lesssim\left|\Delta m_{41}^{2}\right| \lesssim 0.3 \mathrm{eV}^{2}[87]$

In this paper, we perform a detailed analysis of the prospects for the search for a light sterile neutrino over a wide $\Delta m_{41}^{2}$ range, starting from $10^{-5} \mathrm{eV}^{2}$ and going all the way up to $10^{2} \mathrm{eV}^{2}$, using 10 years of atmospheric neutrino data expected from the proposed $50 \mathrm{kt}$ magnetized Iron Calorimeter (ICAL) detector [95] under the India-based Neutrino Observatory (INO) project [96]. The magnetized INO-ICAL detector would detect the atmospheric $\nu_{\mu}$ and $\bar{\nu}_{\mu}$ separately over a wide range of energies and baselines. These features would help the ICAL detector to probe the presence of $\Delta m_{41}^{2}$ over a wide range and also to determine its sign. The charged-current (CC) interactions of $\nu_{\mu}$ and $\bar{\nu}_{\mu}$ inside the ICAL detector will produce $\mu^{-}$and $\mu^{+}$particles, respectively. The ICAL detector would be able to measure the energies and directions of these muons to a very good precision [97]. Moreover, the ICAL detector would be sensitive to neutrinos in the multi-GeV range, and the energies of hadron showers produced by the interactions of these multi-GeV neutrinos can also be well-estimated $[98,99]$. While the main aim of this detector is to resolve the issue of neutrino mass ordering [95, 100-102] and to improve the precision on atmospheric neutrino mixing parameters [95, 101, 103-105], the above features can also be instrumental in the search for a light sterile neutrino.

A sensitivity study of ICAL to a sterile neutrino in the range $0.1 \mathrm{eV}^{2} \lesssim \Delta m_{41}^{2} \lesssim 10 \mathrm{eV}^{2}$ has recently been performed [106], where exclusion limits on the mixing parameters with $500 \mathrm{kt}$-yr exposure have been shown. In this paper, we not only extend this analysis to much lower values of $\Delta m_{41}^{2}$ (all the way down to $10^{-5} \mathrm{eV}^{2}$ ), but also point out for the first time that in the $\Delta m_{41}^{2}$ range of $(0.5-5) \times 10^{-3} \mathrm{eV}^{2}$, ICAL has a far greater sensitivity to the magnitude as well as sign of $\Delta m_{41}^{2}$. We also explore the impact of hadron energy calibration, the actual value of $\left|U_{e 4}\right|$, up-going vs. down-going events, spectral information, and the signs of $\Delta m_{31}^{2}$ as well as $\Delta m_{41}^{2}$, on the sensitivity of ICAL to a sterile neutrino. In addition, in the scenario where a light sterile neutrino exists in Nature, we study the role of ICAL, in particular its muon charge identification capability, in identifying the mass ordering configuration of the four-neutrino spectrum.

We plan this article in the following fashion. We start section 2 with a discussion on different mass ordering schemes and configurations which are possible in the four-neutrino 


\begin{tabular}{|l|c|c|c|c|c|c|c|c|}
\hline Ordering scheme & \multicolumn{3}{|c|}{ N-N } & N-I & \multicolumn{2}{|c|}{ I-N } & \multicolumn{2}{|c|}{ I-I } \\
\hline Sign of $\Delta m_{31}^{2}$ & \multicolumn{3}{|c|}{+} & + & \multicolumn{2}{|c|}{-} & \multicolumn{2}{|c|}{-} \\
Sign of $\Delta m_{41}^{2}$ & \multicolumn{3}{|c|}{+} & - & \multicolumn{2}{|c|}{+} & \multicolumn{2}{c|}{} \\
\hline Sign of $\Delta m_{42}^{2}$ & + & + & - & - & + & - & - & - \\
Sign of $\Delta m_{43}^{2}$ & + & - & - & - & + & + & + & - \\
\hline Configuration & N-N-1 & N-N-2 & N-N-3 & N-I & I-N-1 & I-N-2 & I-I-1 & I-I-2 \\
\hline
\end{tabular}

Table 1. All possible neutrino mass ordering schemes and configurations in the four-neutrino framework.

$(4 \nu)$ framework. Then we describe the lepton mixing matrix in the $4 \nu$ scheme and mention the present constraints that we have on $\left|U_{e 4}\right|^{2},\left|U_{\mu 4}\right|^{2}$, and $\left|U_{\tau 4}\right|^{2}$ from various oscillation experiments. We further elaborate on the Earth matter effects in the presence of three active neutrinos and one light sterile neutrino. We end this section by drawing the neutrino oscillograms in $E_{\nu}-\cos \theta_{\nu}$ plane in the $4 \nu$ paradigm. In section 3, we mention our analysis procedure and present the expected event spectra at the ICAL detector as a function of $E_{\mu}$ and $\cos \theta_{\mu}$ for several benchmark choices of active-sterile oscillation parameters. We identify the regions in $E_{\mu}$ and $\cos \theta_{\mu}$ plane which give significant contributions in constraining active-sterile oscillations. In section 4 , we determine the parameter space in the $\left|\Delta m_{41}^{2}\right|-\left|U_{\mu 4}\right|^{2}$ plane that can be excluded by the ICAL data if there is no light sterile neutrino in Nature. We also study the dependence of these constraints on $\left|U_{e 4}\right|$, mass ordering schemes, as well as energy and direction measurements. In section 5, we assume that there is a light sterile neutrino in Nature and then address several interesting issues, like the precision in the determination of $\Delta m_{41}^{2}$ and the chances of measuring its sign at the INO-ICAL atmospheric neutrino experiment. Finally in section 6, we summarize and draw our conclusions.

\section{Neutrino mixing and oscillations in four flavors}

\subsection{Mass ordering schemes and configurations}

The three active neutrinos $\nu_{e, \mu, \tau}$ and the sterile neutrino $\nu_{s}$ give rise to four mass eigenstates, $\nu_{1,2,3,4}$. Here $\nu_{1,2,3}$ are dominated by active flavors, in the decreasing order of electron flavor fraction, while $\nu_{4}$ is dominated by the sterile neutrino. The mass eigenstates may be arranged, according to their relative mass squared values, in eight possible configurations that are allowed by the current data. We label these configurations initially according to the signs of $\Delta m_{31}^{2}$ and $\Delta m_{41}^{2}(\mathrm{~N}-\mathrm{N}, \mathrm{N}-\mathrm{I}, \mathrm{I}-\mathrm{N}$ and I-I, as shown in the top row of table 1), and further by the relative position of $\nu_{4}$ on the $m_{i}^{2}$-scale, which will depend on the signs of $\Delta m_{42}^{2}$ and $\Delta m_{43}^{2}$, as indicated in the lower rows of table 1 . The configurations have also been shown graphically in figure 1 .

In this paper, we shall focus on exploring these configurations by observing atmospheric neutrinos over a wide range of energies and baselines at INO-ICAL. Some of these configurations, viz. N-N-1 and I-N-1, correspond to the sterile neutrino solution required to satisfy the SBL anomalies discussed in the previous section, in the so-called $3+1$ scheme [107- 

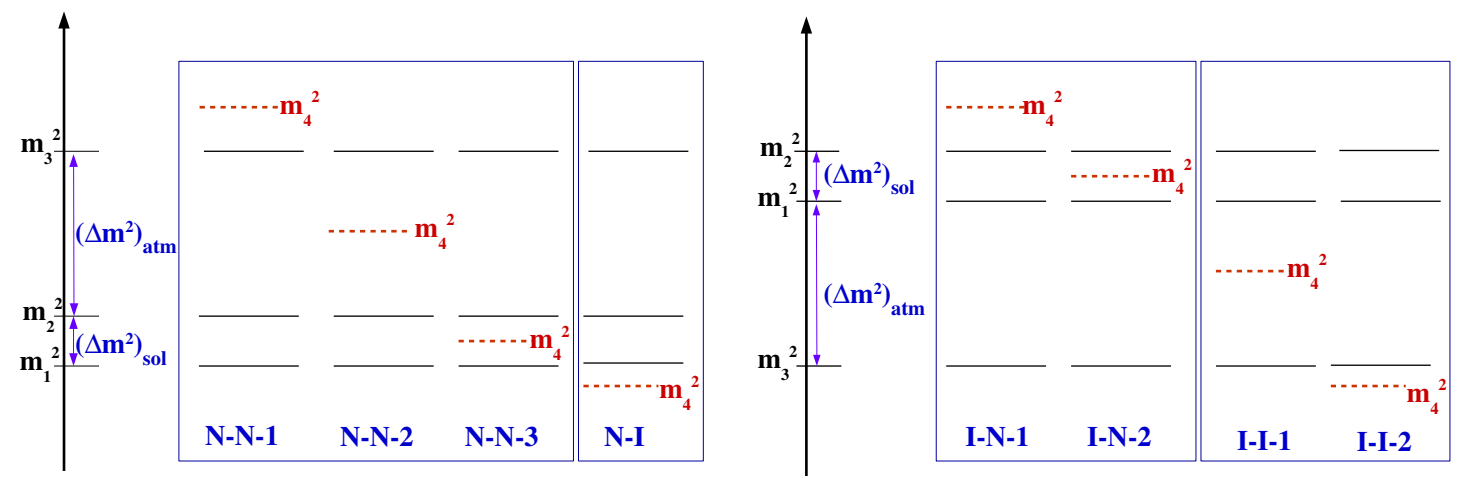

Figure 1. Graphical representations of the mass ordering configurations given in table 1 .

111], while still being consistent with the cosmological bounds on the sum of neutrino masses [112]. Some others, viz. N-N-3 and I-N-2, may be relevant for a super-light sterile neutrino that has been proposed $[78,79,81,82]$ to explain the non-observation of the upturn in the low energy solar data $[11,12,14]$. However, in this work, we shall not be looking for a solution to any of these anomalies, rather we shall perform an agnostic search for a sterile neutrino that may belong to any of these configurations. Note that the configurations N-I and I-I-2 will be disfavoured by the cosmological bounds [112], unless the magnitude of $\Delta m_{41}^{2}$ is very small.

\subsection{The lepton mixing matrix}

In the $4 \nu$ scheme, the four flavor eigenstates $\left(\nu_{e}, \nu_{\mu}, \nu_{\tau}, \nu_{s}\right)$ are linked to the mass eigenstates $\left(\nu_{1}, \nu_{2}, \nu_{3}, \nu_{4}\right)$ via a $4 \times 4$ unitary lepton mixing matrix, which has the following form:

$$
U \equiv\left(\begin{array}{cccc}
U_{e 1} & U_{e 2} & U_{e 3} & U_{e 4} \\
U_{\mu 1} & U_{\mu 2} & U_{\mu 3} & U_{\mu 4} \\
U_{\tau 1} & U_{\tau 2} & U_{\tau 3} & U_{\tau 4} \\
U_{s 1} & U_{s 2} & U_{s 3} & U_{s 4}
\end{array}\right) .
$$

Here, we expect $\left|U_{e 4}\right|^{2},\left|U_{\mu 4}\right|^{2},\left|U_{\tau 4}\right|^{2} \ll 1$, while $\left|U_{s 4}\right|^{2} \approx 1[34,35,51-53]$. There are constraints on active-sterile mixing parameters from atmospheric, long-baseline, as well as reactor experiments.

The Super-Kamiokande Collaboration limits $\left|U_{\mu 4}\right|^{2}$ to less than 0.04 for $\Delta m_{41}^{2}>$ $0.1 \mathrm{eV}^{2}$ at $90 \%$ C.L. in N-N-1 configuration using 4438 live-days of atmospheric neutrino data and assuming $\left|U_{e 4}\right|^{2}=0$ [49]. The IceCube neutrino telescope at the South Pole searches for active-sterile oscillation in their measured atmospheric muon neutrino spectrum as a function of zenith angle and energy in the $320 \mathrm{GeV}-20 \mathrm{TeV}$ range [50]. The muon antineutrinos in their data sample do not experience a strong MSW resonance effect, which allows them to obtain a bound on the active-sterile mixing. As an example, for $\Delta m_{41}^{2} \approx 1.75 \mathrm{eV}^{2}$ (N-N-1 configuration), which is the best-fit value of $\Delta m_{41}^{2}$ according to 


\begin{tabular}{|l|l|l|l|l|l|l|}
\hline Parameter & $\sin ^{2} 2 \theta_{12}$ & $\sin ^{2} \theta_{23}$ & $\sin ^{2} 2 \theta_{13}$ & $\Delta m_{21}^{2}\left(\mathrm{eV}^{2}\right)$ & $\left|\Delta m_{31}^{2}\right|\left(\mathrm{eV}^{2}\right)$ & $\delta_{\mathrm{CP}}$ \\
\hline Value & 0.84 & 0.5 & 0.1 & $7.5 \times 10^{-5}$ & $2.4 \times 10^{-3}$ & $0^{\circ}$ \\
\hline
\end{tabular}

Table 2. Benchmark values of the three-flavor neutrino oscillation parameters considered in this work.

ref. [34], the IceCube data give a bound of $\sin ^{2} 2 \theta_{24} \lesssim 0.06$ at the $90 \%$ C.L. ${ }^{3}$ In the same $\Delta m_{41}^{2}$ range, the MINOS Collaboration places a new upper limit of $\sin ^{2} \theta_{24}<0.03$ at $90 \%$ C.L. using their long-baseline data, suggesting that $\left|U_{\mu 4}\right|^{2} \lesssim 0.03[48,113]$.

The combined analysis of the available data from the reactor experiments Daya Bay and Bugey-3 provides a new upper limit of $\sin ^{2} 2 \theta_{14} \lesssim 0.06$ at $90 \%$ C.L. ${ }^{4}$ around $\Delta m_{41}^{2} \approx$ $1.75 \mathrm{eV}^{2}$ [113]. Recently, the reactor antineutrino experiments NEOS [41] and DANSS [42] have provided new hints in favor of short-baseline $\bar{\nu}_{e}$ oscillations, which are model independent in the sense that these indications are not dependent on the precise estimate of the reactor $\bar{\nu}_{e}$ fluxes. In N-N-1 configuration, the combined analysis of the DANSS and NEOS spectral ratios predict a narrow- $\Delta m_{41}^{2}$ island at $\Delta m_{41}^{2} \approx 1.75 \mathrm{eV}^{2}$ with $\sin ^{2} 2 \theta_{e e}=0.049 \pm 0.023$ at $2 \sigma$ [43], which means that the best-fit value of $\left|U_{e 4}\right|^{2}$ is around 0.012. Another recent study on the same topic can be found in ref. [52].

As far as the constraint on $\left|U_{\tau 4}\right|^{2}$ is concerned, the Super-Kamiokande experiment places an upper limit of 0.18 on this parameter at $90 \%$ C.L., for $\Delta m_{41}^{2}>1 \mathrm{eV}^{2}$ [49]. The IceCube DeepCore Collaboration sets upper bounds of $\left|U_{\mu 4}\right|^{2}<0.11$ and $\left|U_{\tau 4}\right|^{2}<0.15$ at $90 \%$ C.L. for $\Delta m_{41}^{2} \sim 1 \mathrm{eV}^{2}$, using their three years of atmospheric neutrino data in the range 10 to $60 \mathrm{GeV}$ [114]. One can test the mixing between sterile and tau neutrino by looking for a depletion in the neutral-current event rates at the far detector of a longbaseline setup. Both the MINOS [115] and $\mathrm{NO} \nu \mathrm{A}$ [116] experiments place competitive constraints on mixing between sterile and tau neutrinos using this approach. According to the global fit study performed in ref. [35], $\left|U_{\tau 4}\right|^{2} \lesssim 0.014$ at $90 \%$ C.L. for any value of $\Delta m_{41}^{2}$ in N-N-1 configuration. The authors of ref. [34] obtain similar bounds on $\left|U_{\tau 4}\right|^{2}$ around $\Delta m_{41}^{2} \approx 6 \mathrm{eV}^{2}$.

The INO-ICAL experiment will probe a combination of the $\nu_{e} \rightarrow \nu_{\mu}$ and $\nu_{\mu} \rightarrow \nu_{\mu}$ oscillation channels, mainly via the charged-current (CC) interactions that produce muons. The active-sterile mixing elements of relevance here are therefore $U_{e 4}$ and $U_{\mu 4}$ (and to some extent $U_{\tau 4}$, due to Earth matter effects). In order to illustrate the impact of activesterile oscillations for different choices of $\Delta m_{41}^{2}$, we shall be using the benchmark values $\left|U_{e 4}\right|^{2}=0.025,\left|U_{\mu 4}\right|^{2}=0.05$, and $\left|U_{\tau 4}\right|^{2}=0$ while showing our oscillograms and event plots. For most of our sensitivity plots, we shall take $\left|U_{e 4}\right|^{2}=0$, unless otherwise mentioned. We shall also demonstrate later that $\left|U_{e 4}\right|^{2}=0$ yields the most conservative bounds in the $\left(\Delta m_{41}^{2},\left|U_{\mu 4}\right|^{2}\right)$ parameter space. As far as the three-neutrino oscillation parameters are concerned, table 2 shows the benchmark values that we consider in this work.

\footnotetext{
${ }^{3}$ In the short-baseline approximation [109], the effective mixing angle $\sin ^{2} 2 \theta_{24} \equiv 4\left|U_{\mu 4}\right|^{2}\left(1-\left|U_{\mu 4}\right|^{2}\right) \approx$ $4\left|U_{\mu 4}\right|^{2}$, so this bound corresponds to $\left|U_{\mu 4}\right|^{2} \lesssim 0.015$.

${ }^{4}$ In the short-baseline approximation [109], the effective mixing angle, $\sin ^{2} 2 \theta_{14} \equiv 4\left|U_{e 4}\right|^{2}\left(1-\left|U_{e 4}\right|^{2}\right) \approx$ $4\left|U_{e 4}\right|^{2}$, so this bound corresponds to $\left|U_{e 4}\right|^{2} \lesssim 0.015$. Occasionally, $\sin ^{2} 2 \theta_{14}$ is also denoted as $\sin ^{2} 2 \theta_{e e}$.
} 


\subsection{Earth matter effects}

The probability for a neutrino produced with flavor $\alpha$ and energy $E$ to be detected as a neutrino of flavor $\beta$ after traveling a distance $L$ in vacuum can be written in terms of the leptonic mixing matrix elements as $[117,118]$ :

$$
\begin{aligned}
P\left(\nu_{\alpha} \rightarrow \nu_{\beta}\right)= & \delta_{\alpha \beta}-4 \sum_{i>j} \operatorname{Re}\left(U_{\alpha i}^{*} U_{\beta i} U_{\alpha j} U_{\beta j}^{*}\right) \sin ^{2} \Delta_{i j} \\
& +2 \sum_{i>j} \operatorname{Im}\left(U_{\alpha i}^{*} U_{\beta i} U_{\alpha j} U_{\beta j}^{*}\right) \sin 2 \Delta_{i j},
\end{aligned}
$$

where $\Delta_{i j} \equiv \Delta m_{i j}^{2} L /(4 E)$. For antineutrinos, the oscillation probability follows eq. (2.2) with the replacement of $U$ with its complex-conjugate matrix. While propagating through the Earth, the elements of the leptonic mixing matrix change, since the forward scattering of neutrinos on the Earth matter gives rise to effective matter potentials

$$
\begin{aligned}
V_{e s} & =\sqrt{2} G_{F}\left(N_{e}-N_{n} / 2\right) & & \text { between } \nu_{e} \text { and } \nu_{s}, \\
V_{\mu s}=V_{\tau s} & =-\sqrt{2} G_{F} N_{n} / 2 & & \text { between } \nu_{\mu / \tau} \text { and } \nu_{s},
\end{aligned}
$$

in the flavor basis. Here $N_{e}$ is the electron number density and $N_{n}$ is the neutron number density inside the Earth. These modified elements need to be used while computing the neutrino oscillation probabilities through layers of different densities inside the Earth.

As neutrinos start crossing longer distances through the Earth, the Earth matter effects start becoming important, as the relative matter potentials $V_{e s}$ and $V_{\mu s}$ are $\sim \Delta m_{31}^{2} /(2 E)$ for atmospheric neutrinos (e.g. for $E=5 \mathrm{GeV}$ and $L=5000 \mathrm{~km}$ ). As a consequence, for $\Delta m_{41}^{2} \sim \Delta m_{31}^{2}$, the Earth matter affects active-sterile conversion probabilities significantly. In this $\Delta m_{41}^{2}$ region, the similar magnitudes $\Delta m_{41}^{2}$ and $\Delta m_{31}^{2}$ also lead to an interference between the oscillation frequencies governed by them, which will manifest itself in the sensitivity of the ICAL experiment in the $\Delta m_{41}^{2}-\left|U_{\mu 4}\right|^{2}$ plane, as we shall see later in our results. For $\Delta m_{41}^{2} \gg \Delta m_{31}^{2}$, which is the parameter space usually focused on (since it is relevant for explaining the LSND anomaly), matter effects are not very important. However for $\Delta m_{41}^{2} \ll \Delta m_{31}^{2}$, a region of the parameter space that we specially explore, the Earth matter effects would play a major role.

In order to compute the neutrino conversion probabilities in the presence of Earth matter numerically, in both the three-flavor and four-flavor scenarios, we use the GLoBES software $[119,120]$ along with its new physics tools. To take into account the Earth matter effects, we take the Preliminary Reference Earth Model (PREM) profile for the density of the Earth [121], with five density steps (to keep the computation time manageable). For the down-going neutrinos ( $L \lesssim 450 \mathrm{~km}$ ), we take all the neutrinos to be produced at an uniform height of $15 \mathrm{~km}$, and consider them to be travelling through vacuum. For the upward-going neutrinos $(L \gtrsim 450 \mathrm{~km}$ ), we neglect the height of the atmosphere, which is small compared to the total distance travelled. We have checked that these approximations do not affect the oscillation probabilities, and hence the event distribution in energy and zenith angle, to any appreciable extent. 

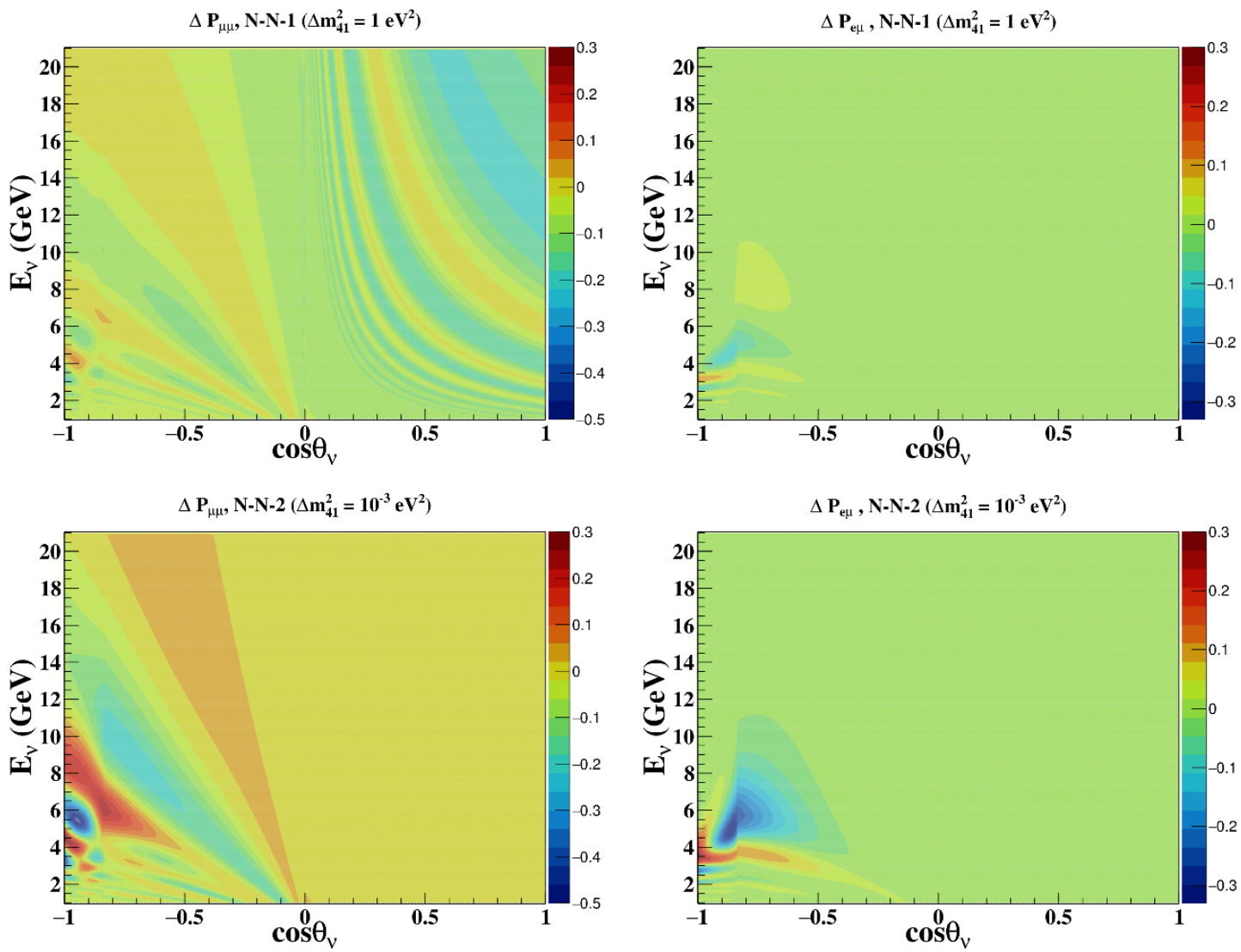

Figure 2. Oscillograms for $\Delta P_{\mu \mu}$ (left panels) and $\Delta P_{e \mu}$ (right panels), and for $\Delta m_{41}^{2}=1 \mathrm{eV}^{2}$ (top panels) and $\Delta m_{41}^{2}=10^{-3} \mathrm{eV}^{2}$ (bottom panels), after performing the averaging over the fast oscillations. We have used $\left|U_{e 4}\right|^{2}=0.0025,\left|U_{\mu 4}\right|^{2}=0.05$, and $\mid U_{\left.\tau 4\right|^{2}}=0$. The hierarchy in the active sector has been taken to be normal $\left(\Delta m_{31}^{2}>0\right)$.

\subsection{Oscillograms in $E_{\nu}-\cos \theta_{\nu}$ plane}

In order to get an idea of the impact of active-sterile mixing on the $\nu_{\mu} \rightarrow \nu_{\mu}$ survival probability $P_{\mu \mu}$ and the $\nu_{e} \rightarrow \nu_{\mu}$ conversion probability $P_{e \mu}$, we define the quantities

$$
\Delta P_{\mu \mu} \equiv P_{\mu \mu}(4 \mathrm{f})-P_{\mu \mu}(3 \mathrm{f}) \quad, \quad \text { and } \quad \Delta P_{e \mu} \equiv P_{e \mu}(4 \mathrm{f})-P_{e \mu}(3 \mathrm{f})
$$

where "(4f)" and "(3f)" denote the quantities calculated in the 4-flavour and 3-flavour mixing scenario, respectively. In figure 2 , we plot these quantities in the $E_{\nu}-\cos \theta_{\nu}$ plane, where $\theta_{\nu}$ is the zenith angle and $E_{\nu}$ is the energy of the neutrino. We present our results for two different values of $\Delta m_{41}^{2}$, viz. $\Delta m_{41}^{2}=1 \mathrm{eV}^{2}$ (corresponding to the N-N-1 configuration), and $\Delta m_{41}^{2}=10^{-3} \mathrm{eV}^{2}$ (corresponding to the $\mathrm{N}-\mathrm{N}-2$ configuration). In the former scenario, we average over the fast oscillations when neutrinos travel large distances through the Earth. From the figure, the following observations may be made:

- Let us first see the impact of the active-sterile mixing in the $\nu_{\mu} \rightarrow \nu_{\mu}$ survival channel, which plays an important role in atmospheric neutrino oscillations. The effect of 
sterile neutrino mixing on $P_{\mu \mu}$ is clearly significant. For $\Delta m_{41}^{2}=1 \mathrm{eV}^{2}$, the effect is observed over a wide region in $E_{\nu}$ and $\cos \theta_{\nu}$. For $\Delta m_{41}^{2}=10^{-3} \mathrm{eV}^{2}$, the impact is mostly confined to the upward going neutrinos, while its magnitude is significant around $E_{\nu} \in[3,10] \mathrm{GeV}$ and $\cos \theta_{\nu} \in[-1,-0.7]$.

- While fast oscillations for the high $\Delta m_{41}^{2} \sim 1 \mathrm{eV}^{2}$ will get averaged out due to the finite energy and angular resolutions of the detector, at $\Delta m_{41}^{2} \sim 10^{-3} \mathrm{eV}^{2}$ the oscillation probability dependence on energy and direction of neutrinos would be more clearly resolvable. Hence we expect that the spectral and angular information should play a role in identifying signatures of sterile neutrinos at low $\Delta m_{41}^{2}$ values .

- The impact of $4 \nu$ mixing on $P_{e \mu}$ is confined to a narrow region to the upwardgoing neutrinos, and it is more prominent at the lower $\Delta m_{41}^{2}$. This would indicate a significant contribution of the matter effects due to the Earth, which influence the upward-going neutrinos.

The much higher effects of active-sterile mixing on $P_{\mu \mu}$, combined with the much higher value of $P_{\mu \mu}$ as compared to $P_{e \mu}$, and the higher fraction of $\nu_{\mu}$ in the atmospheric neutrino flux, indicates that the $\nu_{\mu} \rightarrow \nu_{\mu}$ conversions will play a dominant role in the sensitivity of atmospheric neutrino detectors to sterile neutrinos. The corresponding antineutrino oscillation probability plots show the same features. In fact, the oscillograms at the higher $\Delta m_{41}^{2}$ values are virtually identical with those for neutrinos, shown in the top panels of figure 2. Therefore, ${ }^{5}$ we should be able to interpret most of our results based on $P_{\mu \mu}$.

The oscillograms of $P_{\mu \mu}$ at low $\Delta m_{41}^{2}$ indicate the presence of significant Earth matter effects. This points to the possible sensitivity of the data to the sign of $\Delta m_{41}^{2}$, a point we shall explore further in section 5.2.

\section{Event spectra at ICAL and identifying crucial $\left(E_{\mu}, \cos \theta_{\mu}\right)$ bins}

\subsection{Event distributions in energy and zenith angle}

In order to perform the simulations of the events in INO-ICAL, we generate the events using the same procedure as described in [95, 100, 101]. The NUANCE event generator [122] is used to generate unoscillated atmospheric neutrino events for 1000 years exposure of the $50 \mathrm{kt}$ ICAL, which are later rescaled to the relevant exposure. The oscillations are implemented with the four-flavor probabilities in matter, using the reweighting algorithm, as described in [100]. The energy and direction resolutions of muons and hadrons are used, as per the latest results of the ICAL Collaboration [97, 98]. We estimate the physics reach of ICAL in probing active-sterile oscillation parameters by combining the muon momentum information $\left(E_{\mu}, \cos \theta_{\mu}\right)$ and the hadron energy information $\left(E_{\text {had }}^{\prime} \equiv E_{\nu}-E_{\mu}\right)$ on an eventby-event basis. For this work, it is assumed that the muon and hadron hits can be separated with $100 \%$ efficiency, and that the background and noise are negligible. The events are

\footnotetext{
${ }^{5}$ Note that there are small differences between neutrino and antineutrino oscillograms for $\Delta P_{e \mu}$ at lower $\Delta m^{2}$ for large propagation distances through the Earth matter, however these have a very small impact on our analysis.
} 


\begin{tabular}{|l|c|c|c|c|c|c|}
\hline Case $\left(\Delta m_{41}^{2}\right)$ & Total $\mu^{-}$ & Total $\mu^{+}$ & Down $\mu^{-}$ & Down $\mu^{+}$ & Up $\mu^{-}$ & Up $\mu^{+}$ \\
\hline 3f & 4736 & 2070 & 3108 & 1361 & 1628 & 709 \\
\hline 4f $\left(1 \mathrm{eV}^{2}\right), \mathrm{N}-\mathrm{N}-1$ & 4296 & 1874 & 2817 & 1234 & 1479 & 641 \\
\hline 4f $\left(10^{-3} \mathrm{eV}^{2}\right), \mathrm{N}-\mathrm{N}-2$ & 4612 & 1984 & 3107 & 1361 & 1505 & 623 \\
\hline 4f $\left(10^{-5} \mathrm{eV}^{2}\right), \mathrm{N}-\mathrm{N}-3$ & 4664 & 2046 & 3108 & 1361 & 1557 & 685 \\
\hline
\end{tabular}

Table 3. Number of events for $500 \mathrm{kt}-\mathrm{yr}$ exposure of ICAL, for $E_{\mu} \in[1,11] \mathrm{GeV}$ and $\mathrm{NH}$ in the active sector. We have taken $\left|U_{e 4}\right|^{2}=0.025,\left|U_{\mu 4}\right|^{2}=0.05$, and $\left|U_{\tau 4}\right|^{2}=0$. The information on hadron energy is not used. For sterile neutrinos, the results have been shown for three different $\Delta m_{41}^{2}$ values, corresponding to the $\mathrm{N}-\mathrm{N}-1, \mathrm{~N}-\mathrm{N}-2$ and $\mathrm{N}-\mathrm{N}-3$ configurations, respectively.

binned into 10 uniform $E_{\mu}$ bins in the range $[1,11] \mathrm{GeV}$, and 20 uniform $\cos \theta_{\mu}$ bins in the range $[-1,1]$. The hadrons are binned in $4 E_{\text {had }}^{\prime}$ bins: $1-2 \mathrm{GeV}, 2-4 \mathrm{GeV}, 4-7 \mathrm{GeV}$ and $7-11 \mathrm{GeV}$, as in [101].

Table 3 gives the number of $\mu^{-}$and $\mu^{+}$events, in $3 \nu$ case and with three benchmark values of $\Delta m_{41}^{2}$ for $4 \nu$ configurations. The hierarchy in the active sector has been taken to be normal $\left(\Delta m_{31}^{2}>0\right)$. It may be observed that for $\Delta m_{41}^{2}=1 \mathrm{eV}^{2}$ (N-N-1 configuration), the number of events with $\left|U_{\mu 4}\right|^{2}=0.05$ is about $10 \%$ less than the number with vanishing $\left|U_{\mu 4}\right|$, as expected after averaging over fast $\Delta_{41}$ oscillations. For $\Delta m_{41}^{2}=10^{-3} \mathrm{eV}^{2}(\mathrm{~N}-$ $\mathrm{N}-2$ configuration), the fractional difference in the total number of events with finite vs. vanishing sterile mixing is rather small. However Up/Down ratios of the $\mu^{-}$and $\mu^{+}$ events are different in these two scenarios, since for these $\Delta m_{41}^{2}$ values, the active-sterile oscillation probability will have a nontrivial zenith angle dependence. Thus for low $\Delta m_{41}^{2}$, the information on angular distribution of these events may be useful.

The last row in table 3 shows the scenario where $\Delta m_{41}^{2}$ is extremely small, $\Delta m_{41}^{2}=$ $10^{-5} \mathrm{eV}^{2}$ (N-N-3 configuration). Note that even at this extremely low $\Delta m_{41}^{2}$ value, the effect of oscillations is still visible through the loss in number of events as well as the difference in the ratio of Up/Down events. This may be attributed to the fact that even when $\Delta m_{41}^{2} \rightarrow 0$, active-sterile oscillations may still occur through the frequencies corresponding to $\left|\Delta m_{42}^{2}\right| \sim 10^{-4} \mathrm{eV}^{2}$ and $\left|\Delta m_{43}^{2}\right| \sim 2.4 \times 10^{-3} \mathrm{eV}^{2}$. As a result, the $\Delta m_{41}^{2} \rightarrow 0$ limit in the $4 \nu$ mixing case does not correspond to the decoupling of sterile neutrinos, and limits on active-sterile mixing may still be obtained in this case. In our analysis, we go down to values of $\Delta m_{41}^{2}$ as low as $10^{-5} \mathrm{eV}^{2}$.

Figure 3 shows the distribution of $\mu^{-}$events as a function of $E_{\mu}$, the reconstructed muon energy in two sample $\cos \theta_{\mu}$-bins, where the efficiencies and resolutions of the detector are folded in. Clearly, the number of events should be smaller in the $4 \nu$ mixing scenario as compared to the $3 \nu$ mixing scenario, due to the nonzero $\left|U_{\mu 4}\right|$. The fractional difference is almost uniform over energy for $\Delta m_{41}^{2}=1 \mathrm{eV}^{2}$ (N-N-1 configuration). The absolute difference in the number of events is the largest in the lowest energy bin and decreases at larger energies. This indicates that the low-energy data will contribute more significantly to the identification of sterile mixing. For $\Delta m_{41}^{2}=10^{-3} \mathrm{eV}^{2}$ (N-N-2 configuration), on the other hand, the difference in the number of events as a function of energy is small and 

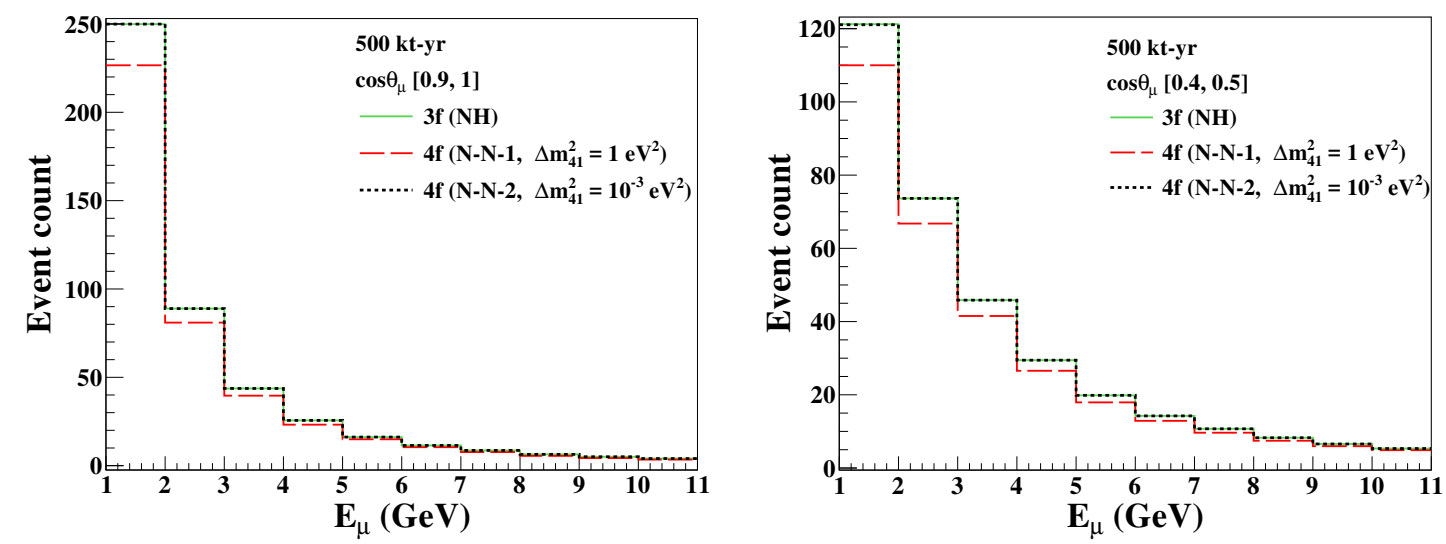

Figure 3. $\mu^{-}$event distributions for $\cos \theta_{\mu} \in[0.9,1.0]$ (left panel) and $\cos \theta_{\mu} \in[0.4,0.5]$ (right panel) in the three-flavor case, and in scenarios with sterile neutrinos of two candidate $\Delta m_{41}^{2}$ values, corresponding to the N-N-1 and N-N-2 configurations. We take $\left|U_{e 4}\right|^{2}=0.025,\left|U_{\mu 4}\right|^{2}=0.05$, and $\left|U_{\tau 4}\right|^{2}=0$. The information on hadron energy is not used. Note that the event distributions in the three-flavour scenario, and in the small- $\Delta m^{2}$ scenario are very close, and their difference cannot be discerned in the figure.
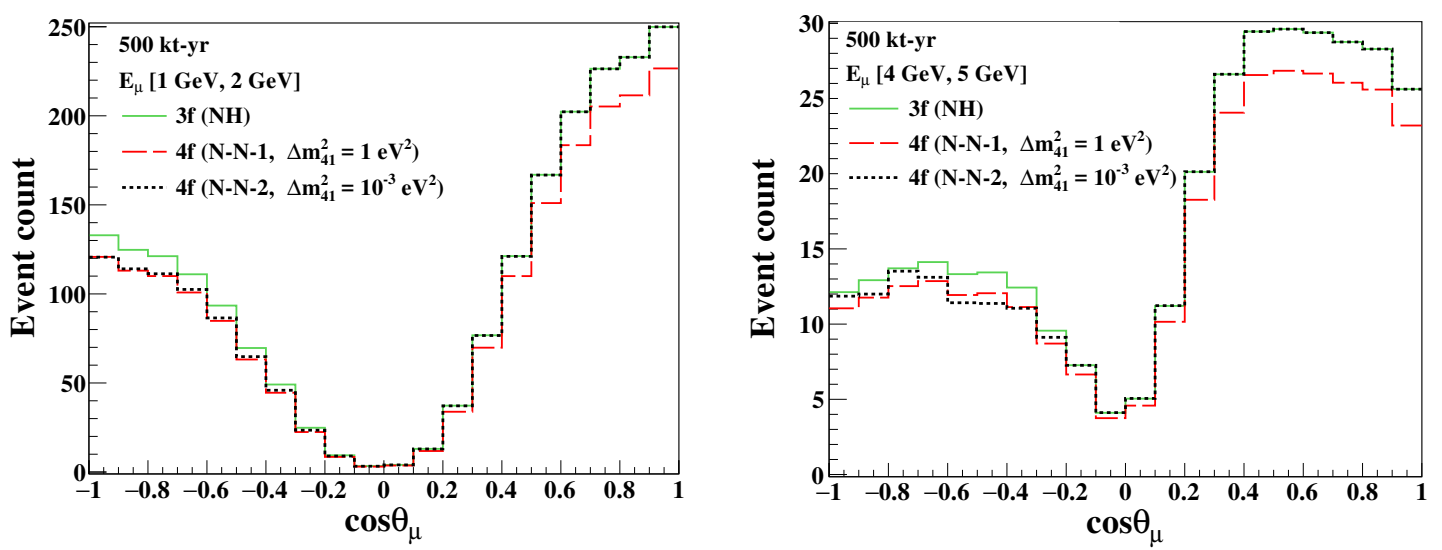

Figure 4. $\mu^{-}$event distributions for $E_{\mu} \in[1,2] \mathrm{GeV}$ (left panel) and $E_{\mu} \in[4,5] \mathrm{GeV}$ (right panel) in the three-flavor case, and in scenarios with sterile neutrinos of two candidate $\Delta m_{41}^{2}$ values, corresponding to the N-N-1 and N-N-2 configurations. We take $\left|U_{e 4}\right|^{2}=0.025,\left|U_{\mu 4}\right|^{2}=0.05$, and $\left|U_{\tau 4}\right|^{2}=0$. The information on hadron energy is not used.

the energy dependence is mild. We have explicitly checked that the features for $\Delta m_{41}^{2}=$ $10^{-5} \mathrm{eV}^{2}$ (N-N-3 configuration) are the same as those for N-N-2 configuration.

Figure 4 shows the event distribution as a function of reconstructed $\cos \theta_{\mu}$ in two sample energy bins. For $\Delta m_{41}^{2}=1 \mathrm{eV}^{2}$ (N-N-1 configuration), the fractional loss in the number of events is observed to be almost uniform throughout the $\cos \theta_{\mu}$ range. On the other hand, $\Delta m_{41}^{2}=10^{-3} \mathrm{eV}^{2}$ (N-N-2 configuration) yields almost no loss of downward-going events $\left(\cos \theta_{\mu} \gtrsim 0\right)$ as compared to the three-flavour scenario, while for the upward-going events $\left(\cos \theta_{\mu} \lesssim 0\right)$, a clear loss of events is observed. This is due to the fact that at such low $\Delta m_{41}^{2}$ values, the downward-going neutrinos do not have enough time to oscillate to the sterile flavor. We have explicitly checked that the features for $\Delta m_{41}^{2}=10^{-5} \mathrm{eV}^{2}(\mathrm{~N}-\mathrm{N}-3$ 
configuration) are the same as those for N-N-2 configuration. This non-trivial dependence of the event spectrum on the direction of the muon is instrumental in providing sensitivity to the value of $\left|U_{\mu 4}\right|$ at low $\Delta m_{41}^{2}$.

\subsection{Useful regions in the $E_{\mu}-\cos \theta_{\mu}$ plane}

We constrain the active-sterile neutrino oscillation by using

$$
\Delta \chi^{2} \equiv \chi^{2}(4 \mathrm{f})-\chi^{2}(3 \mathrm{f})
$$

Here, we define the $\chi^{2}$ for the four-flavor as well as three-flavor scenarios as follows.

First we compute the $\chi_{-}^{2}$ for $\mu^{-}$events. In the "2D" analysis, where the information on only $E_{\mu}$ and $\theta_{\mu}$ is used, we define the $\chi^{2}$ as [123-125]:

$$
\chi_{-(2 \mathrm{D})}^{2}=\min _{\xi_{l}} \sum_{j=1}^{N_{E \mu}} \sum_{k=1}^{N_{\cos \theta_{\mu}}}\left[2\left(N_{j k}^{\text {theory }}-N_{j k}^{\text {data }}\right)-2 N_{j k}^{\text {data }} \ln \left(\frac{N_{j k}^{\text {theory }}}{N_{j k}^{\text {data }}}\right)\right]+\sum_{l=1}^{5} \xi_{l}^{2},
$$

where

$$
N_{j k}^{\text {theory }}=N_{j k}^{0}\left(1+\sum_{l=1}^{5} \pi_{j k}^{l} \xi_{l}\right) .
$$

In eq. (3.2), $N_{j k}^{\text {theory }}$ and $N_{j k}^{\text {data }}$ denote the expected and observed number of $\mu^{-}$events, respectively, in a given $\left(E_{\mu}, \cos \theta_{\mu}\right)$ bin [100]. Here $N_{j k}^{0}$ represents the number of events without systematic errors; we have taken $N_{E_{\mu}}=10$ and $N_{\cos \theta_{\mu}}=20$ as mentioned earlier. The quantities $\xi_{l}$ indicate the "pulls" due to the systematic uncertainties. Following [100], we have included five systematic errors in our analysis: a) flux normalization error (20\%), b) cross section error $(10 \%)$, c) tilt error $(5 \%)$, d) zenith angle error $(5 \%)$, and e) an overall systematics (5\%).

While using the additional information on hadron energy (the so-called "3D" analysis in [101]), the Poissonian $\chi_{-}^{2}$ for $\mu^{-}$events takes the form:

$$
\chi_{-}^{2}(3 \mathrm{D})=\min _{\xi_{l}} \sum_{i=1}^{N_{E_{\text {had }}^{\prime}}} \sum_{j=1}^{N_{E_{\mu}}} \sum_{k=1}^{N_{\cos \theta_{\mu}}}\left[2\left(N_{i j k}^{\text {theory }}-N_{i j k}^{\text {data }}\right)-2 N_{i j k}^{\text {data }} \ln \left(\frac{N_{i j k}^{\text {theory }}}{N_{i j k}^{\text {data }}}\right)\right]+\sum_{l=1}^{5} \xi_{l}^{2},
$$

where

$$
N_{i j k}^{\text {theory }}=N_{i j k}^{0}\left(1+\sum_{l=1}^{5} \pi_{i j k}^{l} \xi_{l}\right)
$$

In eq. (3.4), $N_{i j k}^{\text {theory }}$ and $N_{i j k}^{\text {data }}$ indicate the expected and observed number of $\mu^{-}$events, respectively, in a given $\left(E_{\mu}, \cos \theta_{\mu}, E_{\text {had }}^{\prime}\right)$ bin. $N_{i j k}^{0}$ stands for the number of events without systematic errors. Since we consider four $E_{\text {had }}^{\prime}$ bins, $N_{E_{\text {had }}^{\prime}}=4$.

For both the "2D" and "3D" analyses, the $\chi_{+}^{2}$ for $\mu^{+}$events is computed following the same procedure described above. We add the individual contributions from $\mu^{-}$and $\mu^{+}$ events to obtain the total $\chi^{2}$ in $3 \nu$ and $4 \nu$ schemes. 

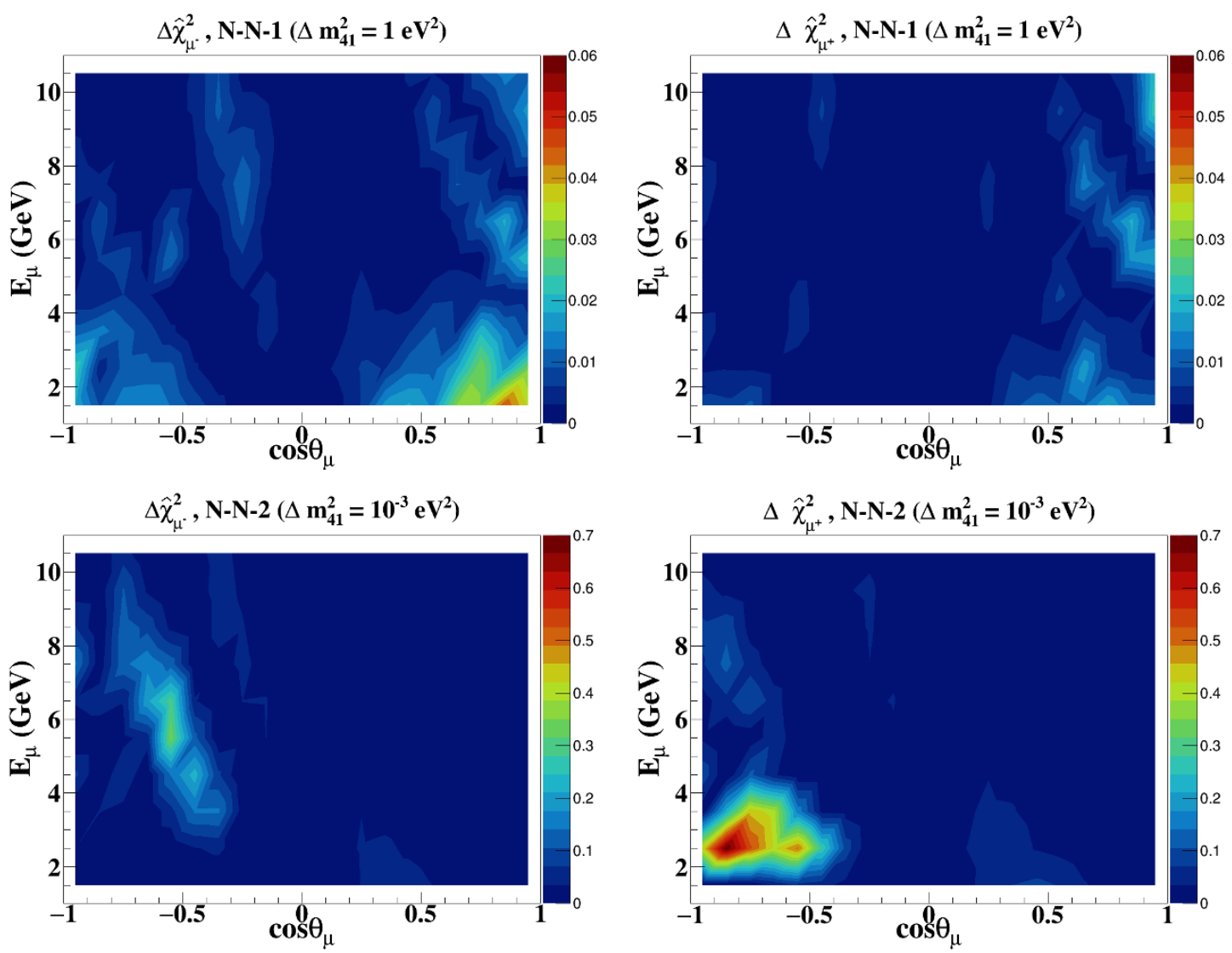

Figure 5. The distributions for $\Delta \widehat{\chi}^{2}$, i.e. $\Delta \chi^{2}$ per energy-cos $\theta$ interval, for $\mu^{-}$(left panel) and $\mu^{+}$(right panel) events generated using $\left|U_{e 4}\right|^{2}=0.025,\left|U_{\mu 4}\right|^{2}=0.05$, and $\left|U_{\tau 4}\right|^{2}=0$. The top panels correspond to $\Delta m_{41}^{2}=1 \mathrm{eV}^{2}$ (N-N-1 configuration), while the bottom panels correspond to $\Delta m_{41}^{2}=10^{-3} \mathrm{eV}^{2}$ (N-N-2 configuration). Only the information on muon energy and direction is used. Note that the scales in the top and bottom panels are different.

In our analysis, the values of the six oscillation parameters of active neutrinos are taken to be fixed, in the simulated data as well as in the fit (see table 2). In order to judge the impact of $\theta_{23}$ and $\Delta m_{31}^{2}$, the two parameters that are expected to influence the atmospheric neutrino measurements strongly, we have also performed the analysis by marginalizing over these two parameters in their current $3 \sigma$ allowed ranges. We observe that, due to the marginalization over $\theta_{23}$ and $\Delta m_{31}^{2}$, the sensitivity of ICAL to sterile neutrino decreases marginally at low $\Delta m_{41}^{2}$, though it remains unchanged at $\Delta m_{41}^{2} \gtrsim 1 \mathrm{eV}^{2}$. Even at lower $\Delta m_{41}^{2}$ values, with the present relative $1 \sigma$ precision of $\sim 5 \%$ on $\sin ^{2} 2 \theta_{13}$ [30-32], the impact of these marginalizations is very small and the results presented in this paper stay valid. The other three parameters that appear in the oscillation probabilities, viz. $\Delta m_{21}^{2}, \theta_{12}$, and $\theta_{13}$, are known to a very good precision, and anyway appear in the atmospheric neutrino oscillation probabilities as sub-leading terms [126]. Also, the impact of $\delta_{\mathrm{CP}}$ in the ICAL experiment is very mild [95].

Before we present our final sensitivity results on active sterile oscillation parameters, we try to locate the regions in $\left(E_{\mu}-\cos \theta_{\mu}\right)$ plane which contribute significantly towards 
$\Delta \chi^{2}$. In figure 5 , we show the distribution of $\Delta \widehat{\chi}^{2}$, i.e. $\Delta \chi^{2}$ per energy-cos $\theta$ interval (as in [101]), in the reconstructed $\left(E_{\mu}-\cos \theta_{\mu}\right)$ plane. Note that for this particular figure, we have not included the effects of the five systematic uncertainties mentioned above. The following insights are obtained from the figure.

- For $\Delta m_{41}^{2}=1 \mathrm{eV}^{2}$ (N-N-1 configuration), we expect there to be a overall suppression, with significant contributions coming from low energy downward going events. This is because at such high values of $\Delta m_{41}^{2}$, active-sterile oscillations develop even from downward neutrinos that travel $\sim 10 \mathrm{~km}$ in the atmosphere, while the active $\nu$ oscillations would not have developed. This feature would be prominent in the $\mu^{-}$events, but not so much in the $\mu^{+}$events due to lack of statistics (See table 3). Overall, we expect there to be an averaged overall suppression of events.

- For $\Delta m_{41}^{2}=10^{-3} \mathrm{eV}^{2}$ (N-N-2 configuration), the information in $\Delta \chi^{2}$ is concentrated in the low-energy upward-going events. This is because at low $\Delta m_{41}^{2}$, it takes $\sim 1000$ $\mathrm{km}$ propagation for the oscillations to develop. Also it may be noticed that the $\Delta \widehat{\chi}^{2}$ in this region is more significant for $\mu^{+}$events. This follows from the effects of the MSW resonance of active-sterile mixing in matter, which appears in the antineutrino channel for the N-N-2 configuration.

- The events around $\cos \theta_{\mu} \sim 0$ do not have much information, since the efficiency of the detector is less for horizontal events [97].

In the next section, we discuss the constraints on the active-sterile mixing parameters in the $\Delta m_{41}^{2}-\left|U_{\mu 4}\right|^{2}$ space, and gain insights into the dependence of these constraints on other mixing parameters like $\left|U_{e 4}\right|$ and the mass ordering configuration.

\section{Constraining active-sterile mixing}

In this section, we present the results on the reach of ICAL for excluding the mixing parameter $\left|U_{\mu 4}\right|^{2}$, as a function of $\Delta m_{41}^{2}$. We generate the data in the absence of sterile neutrino, and try to fit it with the hypothesis of the presence of a sterile neutrino, with $\Delta m_{41}^{2}$ ranging from $10^{2} \mathrm{eV}^{2}$ all the way down to $10^{-5} \mathrm{eV}^{2}$. This range covers, and goes well beyond, the $\Delta m_{41}^{2}$ range relevant for the LSND anomaly on the higher side and the solar $\Delta m_{21}^{2}$ on the lower side. We use an exposure of $500 \mathrm{kt}-\mathrm{yr}$, and present the results in terms of $90 \%$ C.L. (2 d.o.f.) exclusion contours in the $\Delta m_{41}^{2}-\left|U_{\mu 4}\right|^{2}$ plane. To generate the prospective data, we take the values of the active neutrino mixing parameters from table 2 and we keep them fixed in the fit. We have checked that, when the accuracy in the measurement of active mixing parameters expected after 10 years is taken into account (using priors), the variation in the values of these parameters does not affect the results to any significant extent. We take $U_{\tau 4}$ as well as all the phases in the active-sterile mixing to be zero throughout the analysis, both in the simulation and in the fit.

In section 4.1, to begin with we restrict ourselves to $\left|U_{e 4}\right|=0$, and the N-N ordering scheme $\left(\Delta m_{31}^{2}>0\right.$ and $\left.\Delta m_{41}^{2}>0\right)$ for the sake of clarity. In section 4.1.1, we study the 


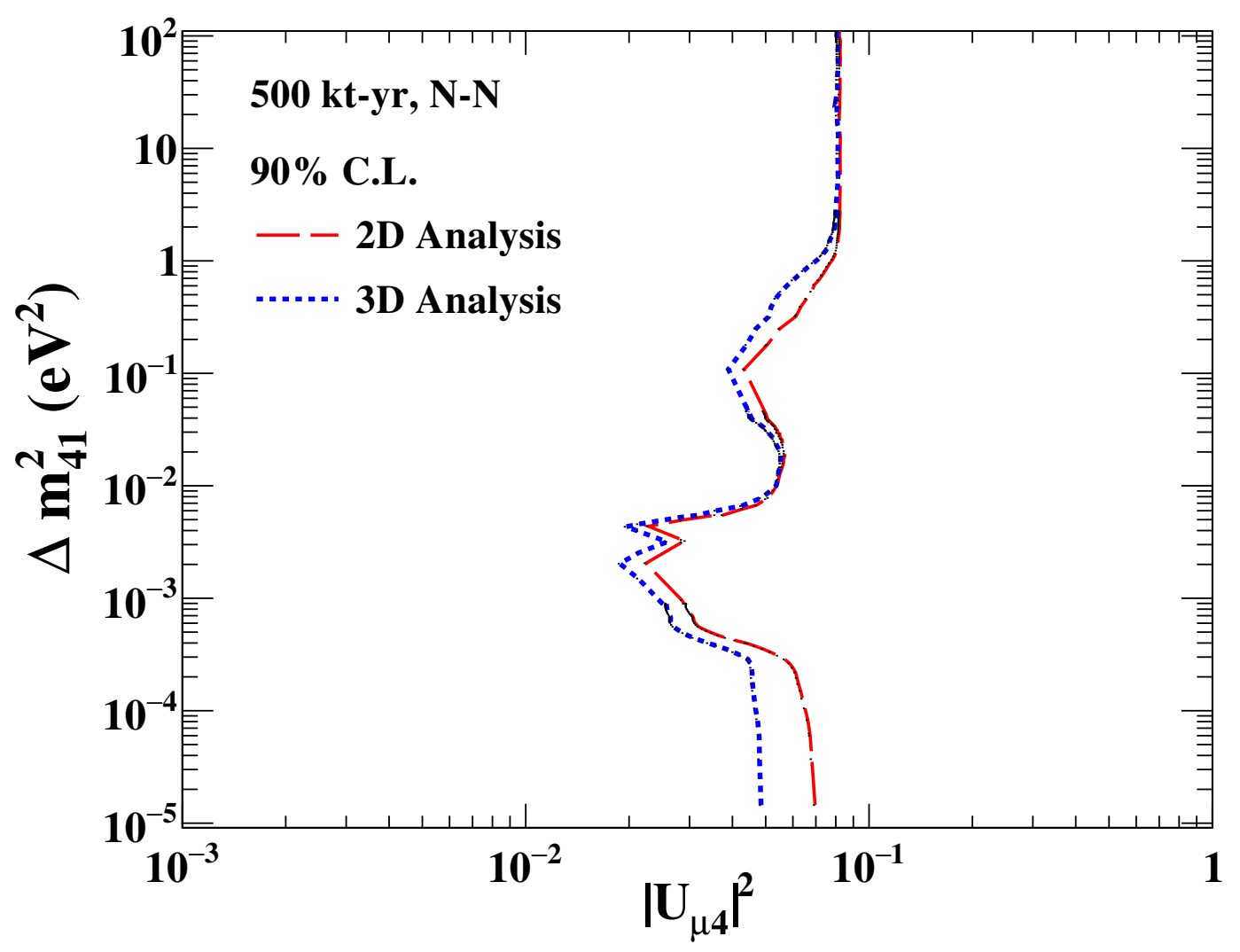

Figure 6. The $90 \%$ C.L. exclusion contours in the $\Delta m_{41}^{2}-\left|U_{\mu 4}\right|^{2}$ plane, with the $2 \mathrm{D}$ analysis (information on muon momentum only), and 3D analysis (inclusion of event-by-event information on hadron energy), for $500 \mathrm{kt}-\mathrm{yr}$ exposure. For illustration, we have used the N-N mass ordering scheme.

dependence of the exclusion contours on nonzero $\left|U_{e 4}\right|$ and find that the bounds in the $\left(\Delta m_{41}^{2},\left|U_{\mu 4}\right|^{2}\right)$ parameter space are the most conservative with $\left|U_{e 4}\right|=0$. We also check the dependence of the results on the mass ordering schemes by computing the exclusion contours for the remaining ordering schemes (N-I, I-N, and I-I), and find it to be very mild. We therefore focus on the N-N ordering scheme and keep $\left|U_{e 4}\right|=0$, in the data as well as in the fit, in the rest of section 4. In section 4.1.2, we study the relative importance of the total rate of events and the shape of their energy as well as angular distributions, and in section 4.2 we compare the INO-ICAL sensitivity in the $\Delta m_{41}^{2}-\left|U_{\mu 4}\right|^{2}$ plane with that of the other ongoing experiments.

\subsection{Exclusion contours in the $\Delta m_{41}^{2}-\left|U_{\mu 4}\right|^{2}$ plane}

Figure 6 shows the exclusion contours obtained using only the muon momentum information (2D analysis), and those obtained by adding the hadron energy information (3D analysis), in the N-N mass ordering scheme. The following features of the reach of ICAL, expressed in terms of the exclusion contours, may be observed and interpreted in terms of broad characteristics of neutrino oscillations. 
- For $\Delta m_{41}^{2} \gtrsim 1 \mathrm{eV}^{2}$, the ICAL can exclude active-sterile mixing for $\left|U_{\mu 4}\right|^{2}>0.08$. The reach of ICAL in this region is independent of the exact value of $\Delta m_{41}^{2}$. This is the high- $\Delta m_{41}^{2}$ region where neutrinos from all directions have undergone many oscillations, such that only the averaged oscillation probability is observable, and there is no $\Delta m_{41}^{2}$-dependence.

- For $10^{-2} \mathrm{eV}^{2} \lesssim \Delta m_{41}^{2} \lesssim 1 \mathrm{eV}^{2}$, the downward-going neutrinos do not have enough time to oscillate, but the upward-going neutrinos do. This non-trivial direction dependence improves the reach of ICAL to $\left|U_{\mu 4}\right|^{2}>0.05$ at $\Delta m_{41}^{2} \sim 10^{-1} \mathrm{eV}^{2}$.

- Our results for $0.1 \mathrm{eV}^{2} \lesssim \Delta m_{41}^{2} \lesssim 10 \mathrm{eV}^{2}$ are consistent with those obtained in [106].

- In the region $10^{-4} \mathrm{eV}^{2} \lesssim \Delta m_{41}^{2} \lesssim 10^{-2} \mathrm{eV}^{2}$, the oscillation frequencies $\widetilde{\Delta}_{41}$ and $\widetilde{\Delta}_{31}$ (where $\sim$ denotes the quantity in the presence of matter) are of the same order of magnitude. As a result, interference effects between these frequencies leads to a further improvement in the reach of ICAL in this parameter range: around $\Delta m_{41}^{2} \sim$ $10^{-3} \mathrm{eV}^{2}$, the ICAL reach can be up to $\left|U_{\mu 4}\right|^{2}>0.03$.

- When $\Delta m_{41}^{2} \lesssim 10^{-4} \mathrm{eV}^{2}$, the active-sterile oscillations due to the frequency $\widetilde{\Delta}_{41}$ will be suppressed. However in this case $\widetilde{\Delta}_{43} \approx-\widetilde{\Delta}_{31}$, and the oscillations due to this frequency will continue to be present. These oscillations will be independent of the value of $\Delta m_{41}^{2}$, and hence the reach of ICAL in this lowest $\Delta m_{41}^{2}$ range, $\left|U_{\mu 4}\right|^{2} \gtrsim 0.07$, would be virtually independent of the exact $\Delta m_{41}^{2}$ value.

- The addition of hadron information, as described in [101], results in a small improvement in the reach of ICAL for excluding $\left|U_{\mu 4}\right|$. The improvement is negligible for $\Delta m_{41}^{2} \gtrsim 1 \mathrm{eV}^{2}$, the region where the $\Delta \chi^{2}$ information is almost uniformly distributed in the whole $E_{\mu^{-}}$and $\cos \theta_{\mu^{-}}$-range, and hence additional details of the hadron information do not make much difference. At lower $\Delta m_{41}^{2}$ values, though, the hadron information may contribute significantly. At the lowest $\Delta m_{41}^{2}$ range, it increases the ICAL reach from $\left|U_{\mu 4}\right|^{2} \gtrsim 0.07$ to $\left|U_{\mu 4}\right|^{2} \gtrsim 0.05$.

\subsubsection{Dependence on $\left|U_{e 4}\right|$ and the mass ordering scheme}

While calculating the exclusion contours in section 4.1, the value of $\left|U_{e 4}\right|$ was taken to be zero. A possible nonzero value of $\left|U_{e 4}\right|$ could change the results. This change may be discerned from the left panel of figure 7 , where we show the change in the exclusion contours when the value of $\left|U_{e 4}\right|^{2}$ is taken to be 0.025 . The reach of ICAL clearly improves with a nonzero $\left|U_{e 4}\right|$. The constraints given in the vanishing- $\left|U_{e 4}\right|$ scenario are thus the most conservative, and in this paper, we continue to give our constraints in this conservative limit. In case the sterile neutrinos are discovered through the oscillations of $\bar{\nu}_{e}$ at reactor experiments, the measured value of $\left|U_{e 4}\right|$ would help in improving the ICAL constraints.

The right panel of figure 7 shows the dependence of the exclusion contours on the mass ordering scheme. The figure suggests that the exclusion reach of ICAL does not depend on the mass ordering scheme for $\Delta m_{41}^{2} \gtrsim 10^{-2} \mathrm{eV}^{2}$, while it would depend mildly on the scheme for $\Delta m_{41}^{2} \lesssim 10^{-2} \mathrm{eV}^{2}$. 

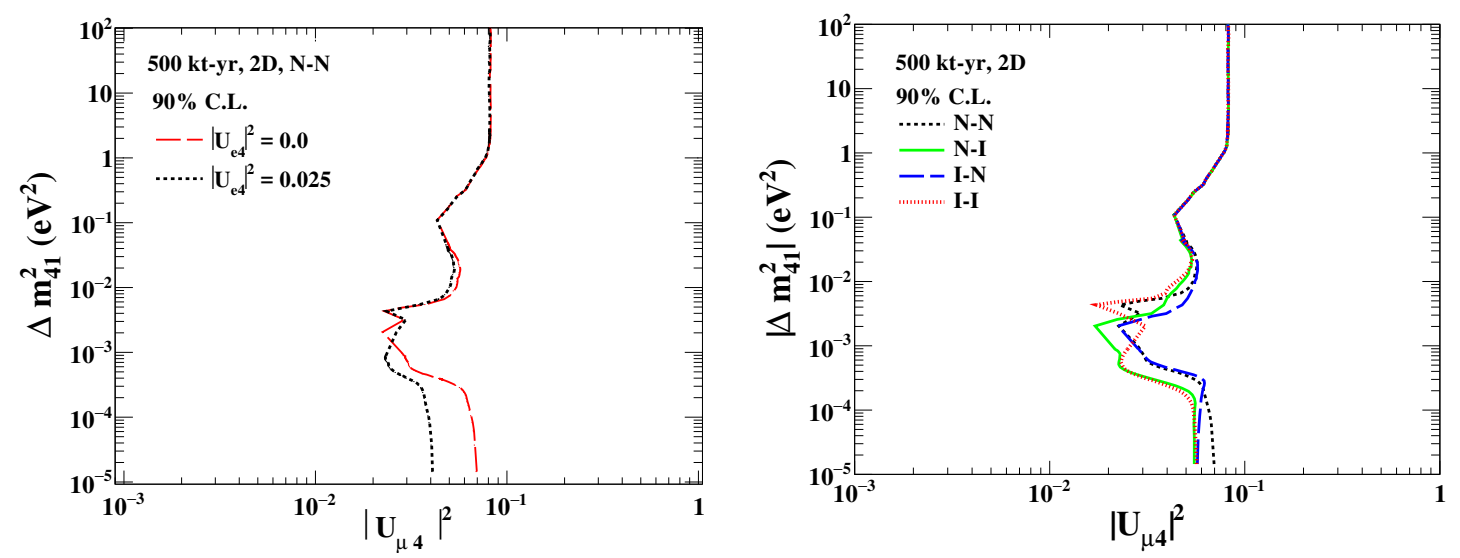

Figure 7. Left panel: the effect of a nonzero value of $\left|U_{e 4}\right|$ on the $90 \%$ exclusion contours, for 500 kt-yr exposure. Right panel: the effect of different mass ordering schemes on the sensitivity, for $\left|U_{e 4}\right|=0$. The results have been presented for the N-N mass ordering scheme, and using only muon information.
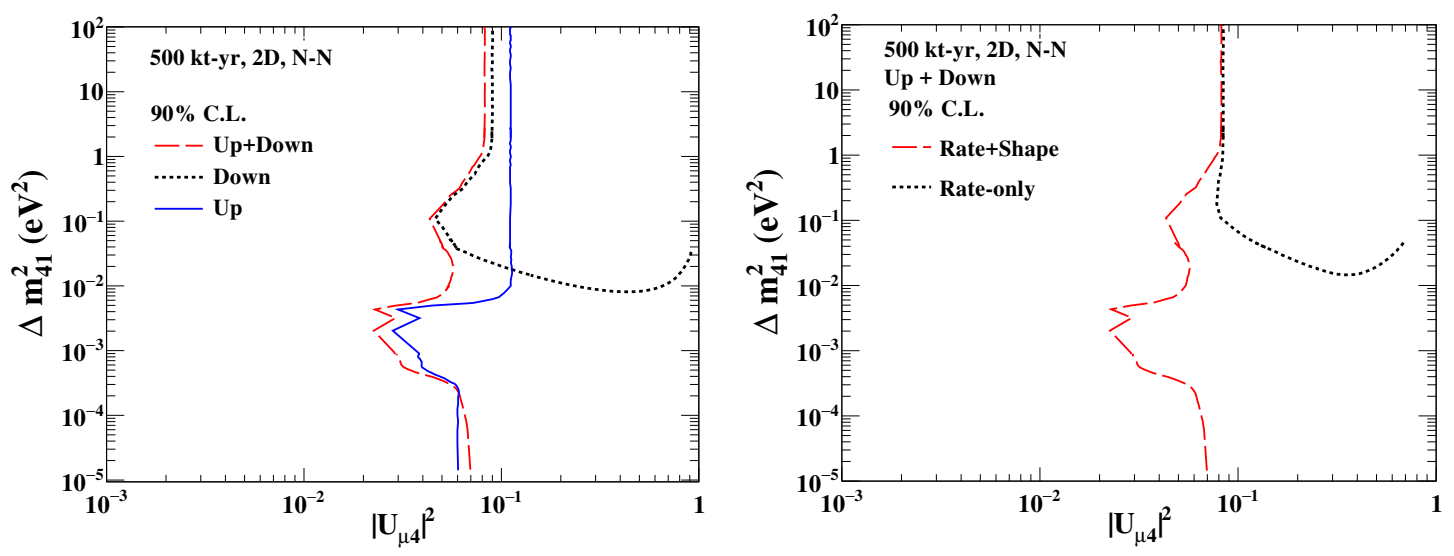

Figure 8. Left panel: $90 \%$ C.L. exclusion regions in the $\left|U_{\mu 4}\right|^{2}-\Delta m_{41}^{2}$ plane with up-going events and down-going events separately, as well as their combined effect. Right panel: $90 \%$ C.L. exclusion regions in the $\left|U_{\mu 4}\right|^{2}-\Delta m_{41}^{2}$ plane using the information on both the rate and shape of the muon spectrum, and using the information only on the rate of muon events (integrated over the range [1-11] GeV and over all the directions). The results have been presented for the N-N mass ordering scheme, and using only muon information.

\subsubsection{Contribution of rate, energy and direction measurements}

The atmospheric data on charged-current muon neutrinos consist of the event rates, as well as the energy and direction of the muon in these events. It is an instructive exercise, and an overall check of our analysis, to compare the relative importance of these quantities, and interpret them in terms of analytical approximations of oscillation probabilities. In figure 8 , we show the exclusion contours if certain information were omitted, which would give us an idea of how important that information is for the analysis.

The left panel of figure 8 indicates the relative importance of upward-going and downward-going events. The right panel of the figure indicates the relative importance of the event rate and the spectral shape (this includes the energy as well as direction information for muons.) The following observations may be made from these figures. 
- For $\Delta m_{41}^{2} \gtrsim 1 \mathrm{eV}^{2}$, the information about the active-sterile mixing is slightly more in the downward-going events than that in the upward-going events. Since we know that in this parameter range the direction of neutrinos does not matter, this difference should be simply due to the larger number of downward-going muon neutrinos (since about half the atmospheric $\nu_{\mu}$ are converted to $\nu_{\tau}$ on their way through the Earth). The reach of ICAL for $\left|U_{\mu 4}\right|^{2}$ values with the downward-going and upward-going data sets shown in the left panel is indeed observed to follow the approximate number of events in this range. This is also consistent with the observation from the right panel, that in this $\Delta m_{41}^{2}$ range all the information is essentially in the event rates, and almost no additional information comes from the energies or directions of the muons.

- For $10^{-2} \mathrm{eV}^{2} \lesssim \Delta m_{41}^{2} \lesssim 1 \mathrm{eV}^{2}$, the left panel shows that the information from upward-going neutrinos is independent of $\Delta m_{41}^{2}$, which may be understood by observing that the upward-going neutrinos in this $\Delta m_{41}^{2}$ range still undergo many oscillations inside the Earth, and only their averaged effect is observed at the detectors. On the other hand, the downward-going neutrinos undergo a small number of oscillations $(\lesssim 1)$ in the atmosphere of the Earth, and hence the exact value of $\Delta m_{41}^{2}$ is relevant. At the higher end of this $\Delta m_{41}^{2}$ range, most of the information about sterile mixing is in the downward-going neutrinos, while at the lower end, the information on sterile oscillations in down-going neutrinos is very small since these neutrinos do not get enough time to oscillate.

- For $10^{-2} \mathrm{eV}^{2} \lesssim \Delta m_{41}^{2} \lesssim 1 \mathrm{eV}^{2}$, the right hand panel shows results that support the above observation. At the lower end of this range, the loss of neutrinos due to oscillations to the sterile species is small, as we have also observed in section 3.1. So the information in only event rates is insignificant. On the other hand, most of the information is now in the neutrino direction (and hence the muon direction).

- At extremely low $\Delta m_{41}^{2}$ values, only the upward-going neutrinos oscillate, and even their number of oscillations is small, so that almost all the information is in the energy and direction distribution of the events.

The analysis in this section shows that the information on muon direction is crucial for $\Delta m_{41}^{2} \lesssim 1 \mathrm{eV}^{2}$. The accurate muon direction measurements at ICAL [95, 97] thus make it a suitable detector for probing low $\Delta m_{41}^{2}$ values.

\subsection{Comparison with other experiments}

Figure 9 shows the projected $90 \%$ C.L. reach of ICAL for $\left|U_{\mu 4}\right|^{2}$ with $500 \mathrm{kt}$-yr of data, and its comparison with the current bounds from the atmospheric neutrino experiments SuperKamiokande [49] and Icecube [50], the short-baseline experiments CCFR [44], MiniBooNE and SciBooNE [47], as well as the long-baseline experiments MINOS and MINOS+ [127].

Super-Kamiokande (SK) is the experiment most similar to the INO-ICAL, in the sense that it is sensitive to the atmospheric neutrinos in a similar energy range. In addition to the $\nu_{\mu} \rightarrow \nu_{\mu}$ and $\nu_{e} \rightarrow \nu_{\mu}$ oscillation channels that ICAL is sensitive to, SK can also detect 


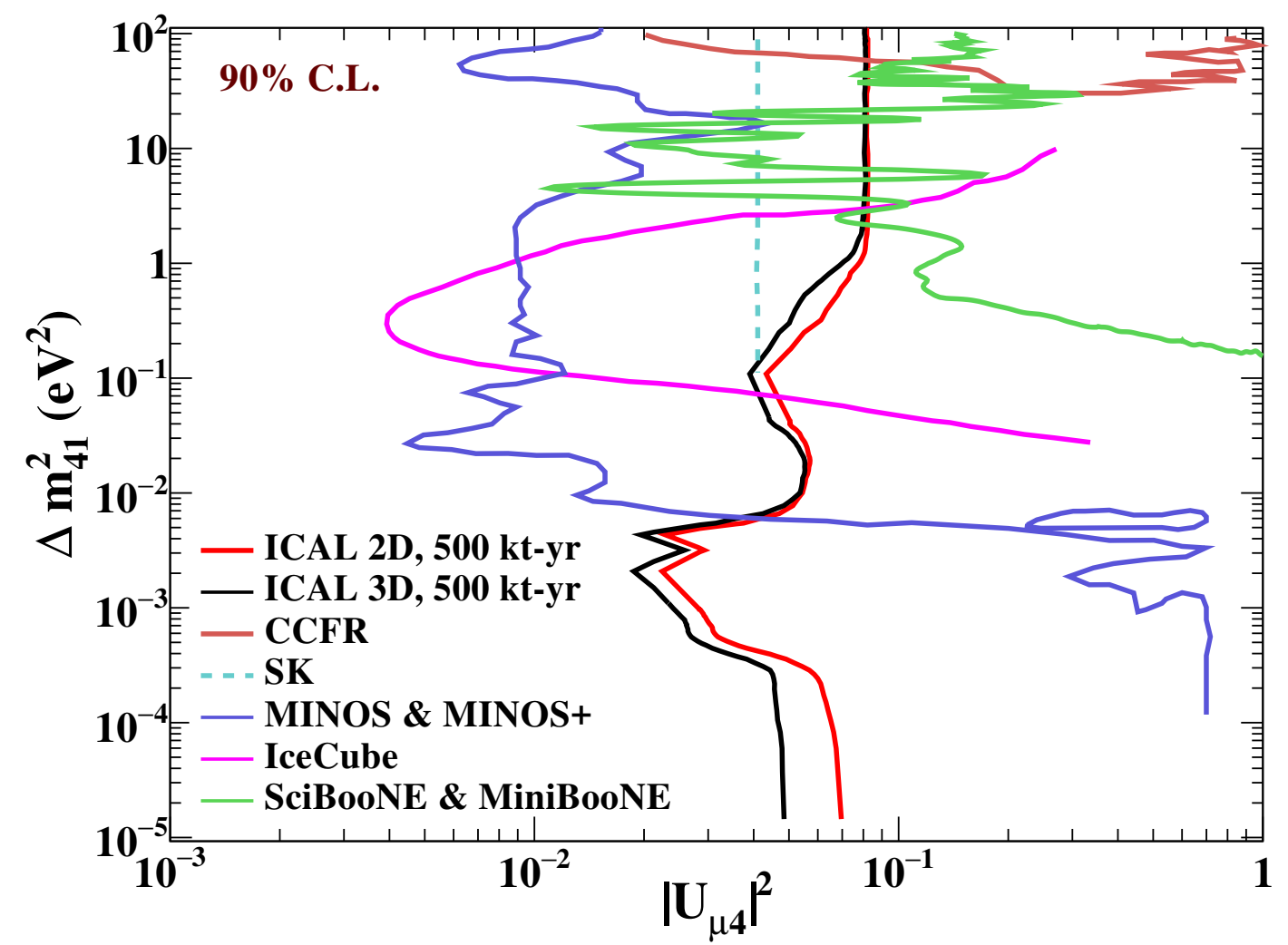

Figure 9. Comparison of the $90 \%$ exclusion contours for INO-ICAL in the N-N mass ordering scheme with an exposure of $500 \mathrm{kt}-\mathrm{yr}$, with the current constraints from other experiments: CCFR [44], Super-Kamiokande (SK) [49], IceCube [50], MINOS and MINOS+ [127], SciBooNE and MiniBooNE [47]. The Super-Kamiokande contour, shown with a dotted line, is the result of the analysis using a one-parameter fit, while all the other contours are with analyses using twoparameter fits.

the charged-current interactions of $\nu_{e}$, and hence can analyze the $\nu_{e} \rightarrow \nu_{e}$ and $\nu_{\mu} \rightarrow \nu_{e}$ channels. (However, in the SK exclusion limits shown in the figure, the information from $\nu_{\mu} \rightarrow \nu_{\mu}$ channel dominates.) It also has a lower energy threshold than ICAL, so it has the advantage of being able to detect the low energy events where the depletion in the number of events is the most prominent for higher $\Delta m_{41}^{2}$ (for example, at $\Delta m_{41}^{2} \gtrsim 1 \mathrm{eV}^{2}$, see figure 3). On the other hand, it cannot distinguish neutrinos from antineutrinos, and does not have as good muon direction resolution as ICAL, which would play a crucial role in identifying sterile neutrinos for lower $\Delta m_{41}^{2}$ values (see figure 4).

An important difference between the sterile neutrino analysis of SK and the other experiments needs to be noted. Since the SK analysis has been performed only for $\Delta m_{41}^{2}>$ $0.1 \mathrm{eV}^{2}$, where the oscillations from all directions are averaged out, the data is not sensitive to the value of $\Delta m_{41}^{2}$. The fit in the analysis is therefore carried out for only one parameter $\left(\left|U_{\mu 4}\right|^{2}\right)$, and therefore the $90 \%$ exclusion contour corresponds to $\Delta \chi^{2}>2.71$. On the other hand, for all the other experiments including ICAL, the $90 \%$ exclusion contours have been evaluated with two-parameter analyses, and hence correspond to $\Delta \chi^{2}>4.61$. If the sensitivity analysis of ICAL were to be performed only in the $\Delta m_{41}^{2}>1 \mathrm{eV}^{2}$ region with 
a single-parameter fit, the $90 \%$ exclusion reach of ICAL would improve from $\left|U_{\mu 4}\right|^{2}>0.08$ to $\left|U_{\mu 4}\right|^{2}>0.06$.

Some of the most stringent bounds on the sterile mixing parameters are given by IceCube [50]. Indeed for $\Delta m_{41}^{2} \approx 0.3 \mathrm{eV}^{2}$, IceCube puts a bound of $\left|U_{\mu 4}\right|^{2} \lesssim 0.004$. However since IceCube has a lower energy threshold of $\sim 100 \mathrm{GeV}$, it is sensitive to only higher values of $\Delta m_{41}^{2} \gtrsim 10^{-2} \mathrm{eV}^{2}$, as is evident from figure 9 .

The fluxes at the fixed-baseline experiments are more well-determined than the atmospheric neutrino fluxes at ICAL. However ICAL has the advantage of a large range of $L / E$, and hence sensitivity to a large range of $\Delta m_{41}^{2}$ values. Indeed, this paper shows how qualitatively different features of oscillation probabilities come into play for different $\Delta m_{41}^{2}$ values, and how ICAL can address all these features so as to be sensitive to a large range of $\Delta m_{41}^{2}$. The CCFR experiment at Fermilab [44] had large neutrino energies $(\gtrsim 100 \mathrm{GeV})$ and a small baseline $(\sim 1 \mathrm{~km})$, and could only probe $\Delta m_{41}^{2} \gtrsim 1 \mathrm{eV}^{2}$. The SciBOONE and MiniBOONE experiments have nearly the same baselines as CCFR, but neutrino energies of $\sim \mathrm{GeV}$, so they could be sensitive to $\Delta m_{41}^{2} \gtrsim 0.1 \mathrm{eV}^{2}$, but no lower [46]. MINOS [127], having a longer baseline of $\sim 735 \mathrm{~km}$, is sensitive to even lower $\Delta m_{41}^{2}$ values, up to $\Delta m_{41}^{2} \gtrsim 10^{-4} \mathrm{eV}^{2}$. At $\Delta m_{41}^{2} \lesssim 10^{-2} \mathrm{eV}^{2}$, ICAL would be one of the few experiments sensitive to active-sterile neutrino mixing.

\section{$5 \quad$ Exploring features of $\Delta m_{41}^{2}$}

In the previous section, we derived the constraints in the $\Delta m_{41}^{2}-\left|U_{\mu 4}\right|^{2}$ space in the scenario where ICAL observes no signal for active-sterile oscillations. In this section, we assume that there is a sterile neutrino with $10^{-5} \mathrm{eV}^{2} \leq \Delta m_{41}^{2} \leq 10^{2} \mathrm{eV}^{2}$, with the mixing parameters $\left|U_{e 4}\right|^{2}=0.025,\left|U_{\mu 4}\right|^{2}=0.05$ and $\left|U_{\tau 4}\right|^{2}=0$. Note that while exploring the features of $\Delta m_{41}^{2}$ in this section, we keep the values of the mixing parameters fixed while generating the data in the $4 \nu$ scenario. Even while fitting the data at test values of $\Delta m_{41}^{2}$, we take the active-sterile mixing parameters to be fixed and known. All the phases in the $4 \nu$ mixing matrix are taken to be zero in the data as well as in the fit. The values of the $3 \nu$ mixing parameters have been kept fixed to the values shown in table 2, and the sign of $\Delta m_{31}^{2}$ has been taken to be positive, while generating the data and performing the fit. In this situation, we attempt to address the following two questions in the context of ICAL for the first time:

- With what precision can we measure a given value of $\Delta m_{41}^{2}$ ?

- Can we identify the sign of $\Delta m_{41}^{2}$ ?

In this process, we will also try to quantify the contributions of two of the unique features of ICAL, viz. charge-identification (CID) capability and the hadron energy measurement on the event-by-event basis.

\subsection{Precision in the determination of $\Delta m_{41}^{2}$}

In order to illustrate the capability of ICAL to measure the value of $\Delta m_{41}^{2}$ precisely, we generate data for an exposure of $500 \mathrm{kt}-\mathrm{yr}$ at $\Delta m_{41}^{2}=10^{-3} \mathrm{eV}^{2}$, which corresponds to the 


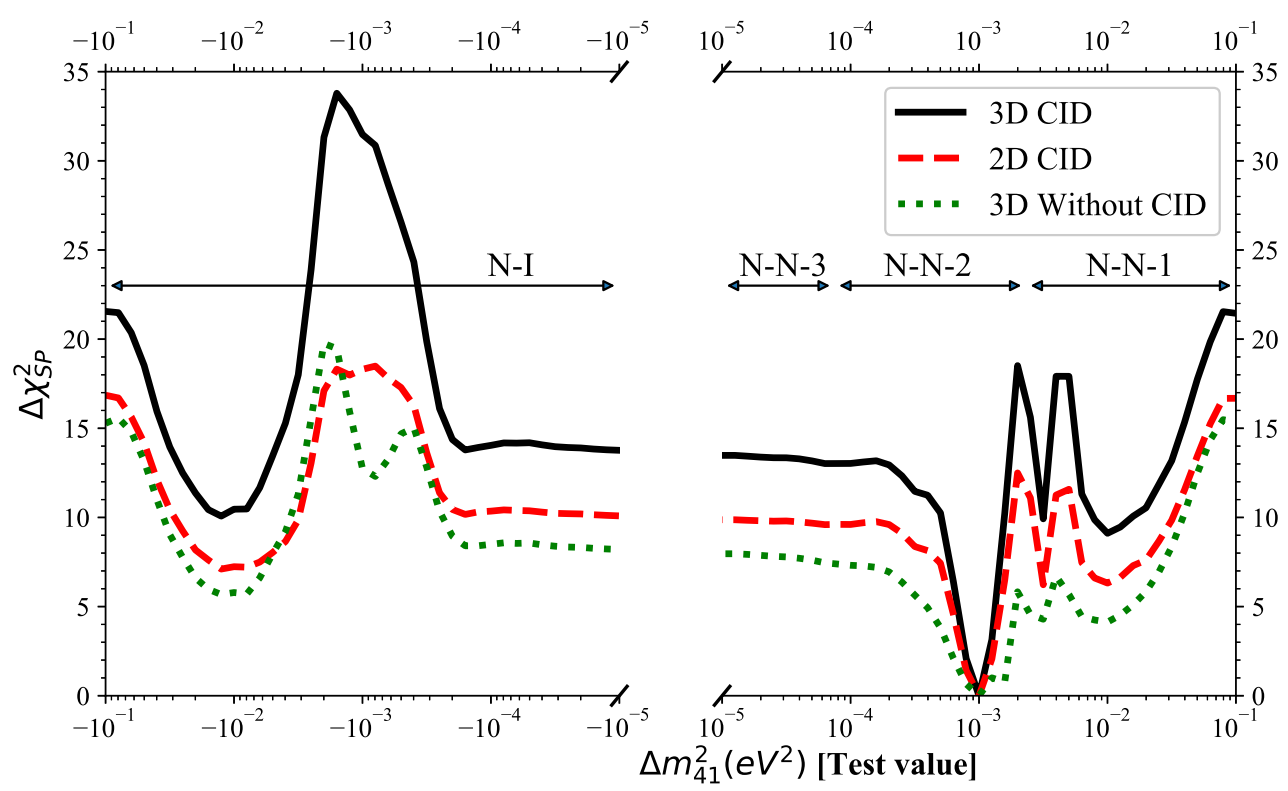

Figure 10. The $\Delta \chi_{\text {SP }}^{2}$ [see eq. (5.1)] at test values of $\Delta m_{41}^{2}$ with $500 \mathrm{kt}$-yr of ICAL data, when the true value is $\Delta m_{41}^{2}=10^{-3} \mathrm{eV}^{2}$ (corresponding to N-N-2). The mixing parameters are taken to be $\left|U_{e 4}\right|^{2}=0.025,\left|U_{\mu 4}\right|^{2}=0.05$, and $\left|U_{\tau 4}\right|^{2}=0$. The results have been presented with and without muon charge identification, and with and without using the hadron energy information.

N-N-2 configuration, The quantity

$$
\Delta \chi_{\mathrm{SP}}^{2}\left[\Delta m_{41}^{2}(\text { test })\right] \equiv \chi^{2}\left[\Delta m_{41}^{2}(\text { test })\right]-\chi^{2}\left[\Delta m_{41}^{2}=10^{-3} \mathrm{eV}^{2}\right]
$$

is then calculated for test values of $\Delta m_{41}^{2}$ in the whole range $10^{-5} \mathrm{eV}^{2}<\Delta m_{41}^{2}<0.1 \mathrm{eV}^{2}$, $-0.1 \mathrm{eV}^{2}<\Delta m_{41}^{2}<-10^{-5} \mathrm{eV}^{2}$, which gives us an idea of how well a particular value of $\Delta m_{41}^{2}$ may be excluded from the data, if indeed there is a sterile neutrino.

The results have been shown in figure 10 for three different cases: (i) 3D CID: information on muon charge identification (CID) as well as hadron energy is used; (ii) 2D CID: information on CID is used, but not on the hadron energy; and (iii) 3D without CID: information on hadron energy is used, but not on CID. The muon momentum information is naturally used in all cases. The comparison of these three cases will help us quantify the importance of hadron energy calibration and muon charge identification, two of the unique features of ICAL. The following observations may be made from this figure.

- The value of $\Delta \chi_{\mathrm{SP}}^{2}$ indeed shows a sharp dip near the true value of $\Delta m_{41}^{2}$. For the benchmark parameter values, it is expected that the other candidate mass ordering configurations, N-N-1, N-N-3 and N-I, could be ruled out at $\gtrsim 3 \sigma$ in the 3D CID case.

- Note that there is a narrow dip in $\Delta \chi_{\mathrm{SP}}^{2}$ near $\Delta m_{41}^{2} \approx(3-4) \times 10^{-3} \mathrm{eV}^{2}$. This is likely to be an effect of the interference between the oscillation frequencies corresponding to $\Delta m_{41}^{2}$ and $\Delta m_{31}^{2}$. 
- It is observed that the values of $\Delta m_{41}^{2} \approx-3 \times 10^{-3}$ would be strongly disfavoured. The reason for this lies in the observation that in such a scenario, there would have been an active-sterile MSW resonance, that would have given rise to significant differences from the actual $\Delta m_{41}^{2} \approx+3 \times 10^{-3}$ scenario, which has no such resonance.

- For $\left|\Delta m_{41}^{2}\right| \gtrsim 10^{-2} \mathrm{eV}^{2}$, the $\Delta \chi_{\mathrm{SP}}^{2}$ values are independent of the sign of $\Delta m_{41}^{2}$, since the active-sterile oscillations get averaged out at such a high magnitude of $\Delta m_{41}^{2}$, and the information about the sign of $\Delta m_{41}^{2}$ is lost.

- In the absence of hadron energy information (2D CID), the value of $\Delta \chi_{\mathrm{SP}}^{2}$ reduces by more than $25 \%$ at almost all test values of $\Delta m_{41}^{2}$. This illustrates the importance of using the hadron energy information, even though the measurement of hadron energy is not very accurate in ICAL.

- Similarly, the absence of muon charge identification (3D without CID) would also reduce $\Delta \chi_{\mathrm{SP}}^{2}$ by more than $25 \%$ at almost all test values of $\Delta m_{41}^{2}$. In particular, it would be difficult to rule out the N-N-3 configuration, or the N-N-1 configuration with $\Delta m_{41}^{2} \lesssim 0.05 \mathrm{eV}^{2}$, to more than $3 \sigma$ if the CID capability were absent. This illustrates the significant advantage ICAL would have due to its excellent muon charge identification.

- The CID capability would also allow us to analyze the $\mu^{-}$and $\mu^{+}$events (equivalently, $\nu_{\mu}$ and $\bar{\nu}_{\mu}$ ) separately, and determine the $\Delta m_{41}^{2}$ values with two independent data sets. This would also serve as a test of CPT violation in the $4 \nu$ framework.

As has been observed above, ICAL may be able to determine $\Delta m_{41}^{2}$ to an accuracy that would enable us to identify the correct $m_{i}^{2}$ configuration from N-N-1, N-N-2, N-N-3, and N-I. In particular, for $\Delta m_{31}^{2}>0$ and $\Delta m_{41}^{2} \approx 10^{-3} \mathrm{eV}^{2}$, it may be able to identify the sign of $\Delta m_{41}^{2}$. In the next section, we explore this possibility further, focusing on the feasibility of such an identification, with true $\Delta m_{41}^{2}$ taken over a wide range.

\subsection{Determination of the sign of $\Delta m_{41}^{2}$}

In the last section, we observed that ICAL is expected to be quite sensitive to the mass ordering configurations in the four-neutrino mass spectrum. Earth matter effects would play a significant role in this identification, since whether the active-sterile resonance takes place in the neutrino or antineutrino channel depends crucially on the sign of $\Delta m_{41}^{2}$. Matter effects thus magnify or suppress the effects due to active-sterile mixing, and make the atmospheric data sensitive to the mass ordering in the sterile sector.

In this section, we shall focus on quantifying the sensitivity of ICAL to the sterile sector mass ordering, i.e. the sign of $\Delta m_{41}^{2}$. Figure 10 already shows that for $\Delta m_{41}^{2}=10^{-3} \mathrm{eV}^{2}$, the wrong mass ordering would be ruled out to $\Delta \chi^{2} \gtrsim 10$ with 500 kt-yr of exposure. We shall now examine how this result depends on the actual value of $\Delta m_{41}^{2}$.

We define the ICAL sensitivity to sterile neutrino hierarchy with the quantity

$$
\Delta \chi_{\mathrm{SH}}^{2} \equiv \chi_{\min }^{2}\left[\Delta m_{41}^{2}(\text { wrong sign })\right]-\chi^{2}\left[\Delta m_{41}^{2}(\text { true })\right]
$$




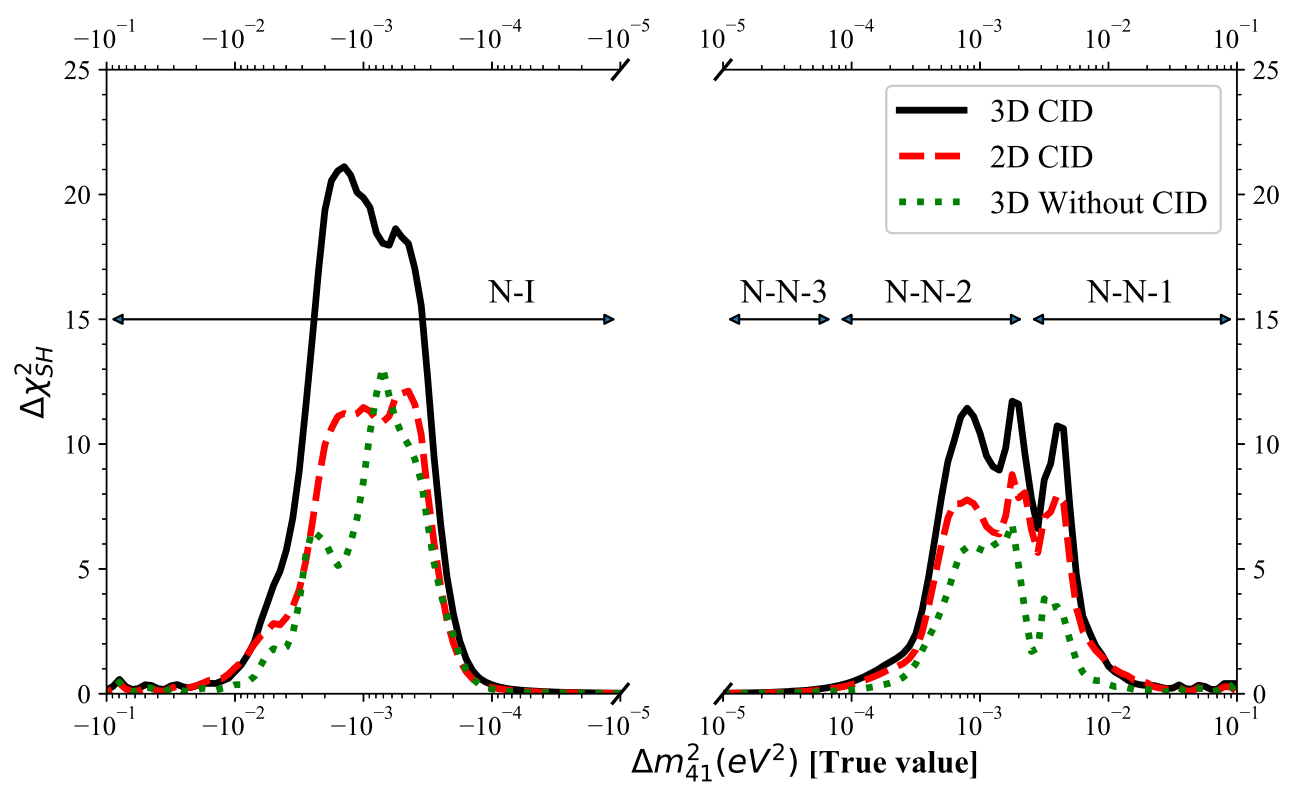

Figure 11. The potential for identification of the sign of $\Delta m_{41}^{2}$ with $500 \mathrm{kt}-\mathrm{yr}$ of ICAL data, quantified by $\Delta \chi_{\mathrm{SH}}^{2}$ [see eq. (5.2)]. The mixing parameters are taken to be $\left|U_{e 4}\right|^{2}=0.025,\left|U_{\mu 4}\right|^{2}=$ 0.05 , and $\left|U_{\tau 4}\right|^{2}=0$. The results have been presented with and without muon charge identification, and with and without using the hadron energy information.

where $\chi_{\min }^{2}\left[\Delta m_{41}^{2}\right.$ (wrong sign) $]$ denotes the minimum value of $\chi^{2}$ when the test value of $\Delta m_{41}^{2}$ is varied over all values with the wrong sign. Note that it is not enough to distinguish $\Delta m_{41}^{2}$ from $-\Delta m_{41}^{2}$, or some other effective value. We would like to ensure that all possible values of $\Delta m_{41}^{2}$ with the wrong sign are excluded.

The results are shown in figure 11 for the three cases 3D CID, 2D CID, and 3D without CID, as earlier. We may observe the following.

- For $\left|\Delta m_{41}^{2}\right| \gtrsim 10^{-2} \mathrm{eV}^{2}$, there is no sensitivity to the sterile hierarchy. This is expected, since the matter effects are negligible for such large values of $\Delta m_{41}^{2}$

- For $\Delta m_{41}^{2} \lesssim 10^{-4} \mathrm{eV}^{2}$, there is no sensitivity to the sterile hierarchy. This may be attributed to the insensitivity of the data to extremely low values of $\Delta m_{41}^{2}$.

- In the intermediate regime $10^{-4} \mathrm{eV}^{2} \lesssim\left|\Delta m_{41}^{2}\right| \lesssim 10^{-2} \mathrm{eV}^{2}$, ICAL is sensitive to the sign of $\Delta m_{41}^{2}$. The sensitivity grows as the value of $\left|\Delta m_{41}^{2}\right|$ gets closer to $10^{-3} \mathrm{eV}^{2}$. This is because at these values of $\Delta m_{41}^{2}$, there is a $\nu_{\mu}-\nu_{s}\left(\bar{\nu}_{\mu}-\bar{\nu}_{s}\right)$ resonance inside the Earth due to MSW effects, for $\Delta m_{41}^{2}<0\left(\Delta m_{41}^{2}>0\right)$. As a result, the neutrino oscillation probabilities will be widely different with the two signs of $\Delta m_{41}^{2}$.

- In the same intermediate range, three sources contribute to the oscillation probabilities: the oscillation frequencies corresponding to $\Delta m_{31}^{2}$ and $\Delta m_{41}^{2}$, as well as the matter potential. The contributions from these sources are of the same order of magnitude in this parameter range. The interference of these three contributions results in the smaller peaks and valleys as observed in the figure. 
- In addition, it is observed that for $\Delta m_{41}^{2}<0$, the $\Delta \chi_{\mathrm{SH}}^{2}$ values are much larger than those for $\Delta m_{41}^{2}>0$. This may be attributed to the facts that the MSW resonance occurs in the $\nu_{\mu}-\nu_{s}$ channel for $\Delta m_{41}^{2}<0$, and the number of neutrino events at ICAL are almost twice the number of antineutrino events.

- In the absence of hadron energy information (2D CID), the $\Delta \chi_{\mathrm{SH}}^{2}$ values reduce by more than $25 \%$, as compared to the $3 \mathrm{D}$ CID case, in the intermediate range. This illustrates the virtue of using the hadron energy information, even though the measurement of hadron energy is not very precise at ICAL.

- Similarly, the absence of CID (3D without CID) would also reduce $\Delta \chi_{\mathrm{SH}}^{2}$ by more than $25 \%$, as compared to the 3D CID case, in the intermediate range. This illustrates the advantage ICAL would have due to its excellent CID.

The above analysis reinforces the expectation that in the range $\left|\Delta m_{41}^{2}\right|=(0.5-5) \times$ $10^{-3} \mathrm{eV}^{2}$, the ICAL detector, due to its capacity to estimate the hadron energy and to determine the muon charge, would be highly sensitive to the sterile mass hierarchy.

\section{$6 \quad$ Summary and concluding remarks}

The proposed ICAL detector in INO will be able to measure the energy and direction of muons, induced by the interactions of atmospheric $\nu_{\mu} / \bar{\nu}_{\mu}$ in the detector, to a very good precision. It will also be able to determine the muon charge, hence distinguish the incoming muon neutrinos from antineutrinos. Its ability to reconstruct the energy of hadron showers on an event-by-event basis will help it observe neutrinos at multi-GeV energies efficiently. All these features help ICAL to be sensitive to the oscillations of atmospheric neutrinos, and to explore the Earth matter effects on them.

In this paper, we explore the sensitivity of the ICAL detector to the active-sterile neutrino mixing parameters, in the presence of a single light sterile neutrino. If we can confirm the existence of such a light sterile neutrino, it would be a revolution in our understanding of neutrinos, as vital as the discovery of $3 \nu$ flavor oscillation. There is no doubt that exploring the properties of such a light sterile neutrino would provide crucial information on the new physics that is being looked for, at the terrestrial neutrino oscillation experiments as well as in astrophysics and cosmology. It is therefore important to explore the sterile neutrino parameter space over a wide range of $\Delta m_{41}^{2}$. The large $L / E$ range scanned by the atmospheric neutrinos makes ICAL sensitive to $\Delta m_{41}^{2}$ even as low as $10^{-5} \mathrm{eV}^{2}$.

An important ingredient of this $4 \nu$ scenario is the relative mass squared values, $m_{i}^{2}$ 's, of the four neutrino mass eigenstates, i.e. the neutrino mass ordering. While $\Delta m_{21}^{2}>0$, the signs of $\Delta m_{31}^{2}$ and $\Delta m_{41}^{2}$ lead to four possible mass ordering schemes (N-N, N-I, I-N, and I-I). Since we would like to explore a wide range of $\Delta m_{41}^{2}$ values, we have eight possible mass ordering configurations (that are the subsets of the above four schemes): N-N-1, N-N2, N-N-3, N-I, I-N-1, I-N-2, I-I-1, I-I-2. One of the main themes in this paper is to quantify the sensitivity of ICAL to the active-sterile mixing parameters, for these mass ordering configurations. 
Our analysis is based on realistic energy and angular resolutions and efficiencies for muons induced in CC $\nu_{\mu}$ interactions, as obtained by the ICAL collaboration through detector simulations. The oscillation channels responsible for the signals at ICAL are $\nu_{\mu} \rightarrow \nu_{\mu}$ and $\bar{\nu}_{\mu} \rightarrow \bar{\nu}_{\mu}$, and to a smaller extent, $\nu_{e} \rightarrow \nu_{\mu}$ and $\bar{\nu}_{e} \rightarrow \bar{\nu}_{\mu}$. The atmospheric neutrino data will therefore be sensitive mainly to the mixing parameter $\left|U_{\mu 4}\right|^{2}$ (which affects all the channels above), with a sub-leading sensitivity to $\left|U_{e 4}\right|^{2}$ (which only affects the appearance channels). ${ }^{6}$ We explore the sensitivity of ICAL to the parameters $\Delta m_{41}^{2}$ and $\left|U_{\mu 4}\right|^{2}$, while pointing out the changes due to the sub-leading contributions from $\left|U_{e 4}\right|^{2}$. In order to achieve this, we solve the neutrino evolution equation in the four-neutrino framework in the presence of Earth matter, using the GLoBES package.

In the scenario where no sterile neutrino is present, we present our sensitivity results in terms of exclusion contours in the $\Delta m_{41}^{2}-\left|U_{\mu 4}\right|^{2}$ plane, for an exposure of $500 \mathrm{kt}-\mathrm{yr}$. It is found that the sensitivity reach of ICAL at very high and very low values of $\Delta m_{41}^{2}$ is independent of $\Delta m_{41}^{2}$. For $\Delta m_{41}^{2} \gtrsim 1 \mathrm{eV}^{2}$, it is due to the averaging of oscillations; while for $\Delta m_{41}^{2} \lesssim 10^{-4} \mathrm{eV}^{2}$, it is because the oscillations are governed by $\left|\Delta m_{43}^{2}\right| \approx\left|\Delta m_{\text {atm }}^{2}\right|$. In these two ranges, ICAL can exclude the active-sterile neutrino mixing $\left|U_{\mu 4}\right|^{2}$ up to 0.08 and 0.05 , respectively, at $90 \%$ C.L. with the two-parameter fit in the 3D analysis mode. For intermediate $\Delta m_{41}^{2}$ values, the shapes of exclusion contours show more interesting features, which are governed by the matter effects and the interference between atmospheric and sterile mass-squared differences. Here the sensitivity is enhanced: for $\Delta m_{41}^{2} \sim 10^{-3} \mathrm{eV}^{2}$, values of $\left|U_{\mu 4}\right|^{2}>0.02$ can be excluded. In the range $\Delta m_{41}^{2} \lesssim 0.5 \times 10^{-3} \mathrm{eV}^{2}$, ICAL can place competitive constraints on $\left|U_{\mu 4}\right|^{2}$ compared to other existing experiments. The exclusion reach is found to be rather insensitive to the value of $\left|U_{e 4}\right|^{2}$ for $\Delta m_{41}^{2} \gtrsim 10^{-3} \mathrm{eV}^{2}$, while at lower $\Delta m_{41}^{2}$, nonzero $\left|U_{e 4}\right|^{2}$ increases the sensitivity reach of ICAL for $\left|U_{\mu 4}\right|^{2}$.

We also explore the effects of using only the up(down)-going muon event samples to identify which kind of events primarily contribute to the ICAL sensitivity in different $\Delta m_{41}^{2}$ regions. As expected, the sensitivity for $\Delta m_{41}^{2} \gtrsim 1 \mathrm{eV}^{2}$ is dominated by the down-going events, where neutrinos oscillate even over short distances. For low $\Delta m_{41}^{2} \lesssim 10^{-2} \mathrm{eV}^{2}$, the sensitivity is dominated by the up-going events. We also find that at high $\Delta m_{41}^{2}$, the event rate information is sufficient to achieve the optimal sensitivity, whereas at low $\Delta m_{41}^{2}$, the sensitivity primarily comes from the shape+rate analysis. Including the hadron energy information in the $\chi^{2}$ analysis also helps to improve the sensitivity marginally. It is observed that the sensitivity reach is more or less independent of the mass ordering scheme assumed (N-N, N-I, I-N or I-I).

If a sterile neutrino exists, then the muon charge identification and hadron energy estimation in ICAL help in the precision measurement of $\Delta m_{41}^{2}$, and even the identification of its sign. We quantify the performance of ICAL in addressing the above important issues for the first time in this paper. For a given true $\Delta m_{41}^{2}=10^{-3} \mathrm{eV}^{2}$, we explicitly calculate the extent to which test values of $\Delta m_{41}^{2}$ can be excluded. We find that the mass ordering configurations N-N-1, N-N-3, and N-I can be ruled out at $\gtrsim 3 \sigma$ for the benchmark parameter values. Since the identification of the mass ordering in the sterile sector would

\footnotetext{
${ }^{6}$ There will also be some sensitivity to $\left|U_{\tau 4}\right|^{2}$ due to the Earth matter effects.
} 
be one of the priorities of any neutrino physics program once a sterile neutrino is discovered, we explore the capability of ICAL to perform this task over a wide range of possible $\Delta m_{41}^{2}$ values. We find that for $\Delta m_{41}^{2} \approx(0.5-5.0) \times 10^{-3} \mathrm{eV}^{2}$, ICAL has a significant capability for identifying the right mass ordering configuration in the sterile sector.

In summary, ICAL has unique strengths which can play an important role in addressing some of the pressing issues in active-sterile oscillations, involving a light sterile neutrino over a wide mass-squared range. It is one of the few experiments sensitive to $\Delta m_{41}^{2} \lesssim 10^{-3} \mathrm{eV}^{2}$ and can put strong limits on $\left|U_{\mu 4}\right|^{2}$ in this range. Its muon charge identification and hadron energy estimation capabilities also help in pinning down the magnitude and sign of $\Delta m_{41}^{2}$ in the range $(0.5-5.0) \times 10^{-3} \mathrm{eV}^{2}$. Note that the results obtained in this paper assume muon and hadron separation with $100 \%$ efficiency and neglect any background. These issues are being addressed by the INO-ICAL Collaboration currently. We hope that the analysis performed in this paper will take ICAL a step forward in its quest to look for new physics beyond the three-neutrino oscillations.

\section{Acknowledgments}

This work is a part of the ongoing effort of INO-ICAL collaboration to study various physics potentials of the proposed ICAL detector. Many members of the collaboration have contributed for the completion of this work, especially those who are part of the Physics Analysis meetings. We are very grateful to K. Bhattacharya, G. Majumder, and A. Redij for their developmental work on the ICAL detector simulation package. We acknowledge the help of J. Kopp for using the new add-on tools for the GLoBES software, and S.S. Chatterjee for helpful discussions on the $3 \nu$ and $4 \nu$ oscillation probabilities. S.K.A. would like to thank A. Smirnov and A. De Gouvea for useful discussions. S.K.A. acknowledges the support from DST/INSPIRE Research Grant [IFA-PH-12], Department of Science and Technology, India and the Young Scientist Project [INSA/SP/YSP/144/2017/1578] from the Indian National Science Academy. M.M.D. acknowledges the support from the Department of Atomic Energy (DAE) and the Department of Science and Technology (DST), Government of India, and the hospitality of the Weizmann Institute of Science, Israel. A.D. acknowledges partial support from the European Union's Horizon 2020 research and innovation programme under the Marie-Sklodowska-Curie grant agreement Nos. 674896 and 690575. T.T. acknowledges support from the Ministerio de Economía y Competitividad (MINECO): Plan Estatal de Investigación (ref. FPA2015- 65150-C3-1-P, MINECO/FEDER), Severo Ochoa Centre of Excellence and MultiDark Consolider (MINECO), and Prometeo program (Generalitat Valenciana), Spain.

Open Access. This article is distributed under the terms of the Creative Commons Attribution License (CC-BY 4.0), which permits any use, distribution and reproduction in any medium, provided the original author(s) and source are credited.

\section{References}

[1] R.N. Mohapatra et al., Theory of neutrinos: a white paper, Rept. Prog. Phys. 70 (2007) 1757 [hep-ph/0510213] [INSPIRE]. 
[2] A. Strumia and F. Vissani, Neutrino masses and mixings and..., hep-ph/0606054 [INSPIRE].

[3] M.C. Gonzalez-Garcia and M. Maltoni, Phenomenology with massive neutrinos, Phys. Rept. 460 (2008) 1 [arXiv:0704.1800] [INSPIRE].

[4] Particle Data Group collaboration, C. Patrignani et al., Review of particle physics, Chin. Phys. C 40 (2016) 100001 [inSPIRE].

[5] B.T. Cleveland et al., Measurement of the solar electron neutrino flux with the Homestake chlorine detector, Astrophys. J. 496 (1998) 505 [INSPIRE].

[6] F. Kaether et al., Reanalysis of the GALLEX solar neutrino flux and source experiments, Phys. Lett. B 685 (2010) 47 [arXiv:1001.2731] [INSPIRE].

[7] SAGE collaboration, J.N. Abdurashitov et al., Measurement of the solar neutrino capture rate with gallium metal. III: Results for the 2002-2007 data-taking period, Phys. Rev. C 80 (2009) 015807 [arXiv:0901.2200] [INSPIRE].

[8] Super-Kamiokande collaboration, J. Hosaka et al., Solar neutrino measurements in Super-Kamiokande-I, Phys. Rev. D 73 (2006) 112001 [hep-ex/0508053] [InSPIRE].

[9] Super-Kamiokande collaboration, J.P. Cravens et al., Solar neutrino measurements in Super-Kamiokande-II, Phys. Rev. D 78 (2008) 032002 [arXiv:0803.4312] [INSPIRE].

[10] Super-Kamiokande collaboration, K. Abe et al., Solar neutrino results in Super-Kamiokande-III, Phys. Rev. D 83 (2011) 052010 [arXiv:1010.0118] [InSPIRE].

[11] Super-Kamiokande collaboration, K. Abe et al., Solar neutrino measurements in Super-Kamiokande-IV, Phys. Rev. D 94 (2016) 052010 [arXiv: 1606. 07538] [InSPIRE].

[12] SNO collaboration, B. Aharmim et al., Combined analysis of all three phases of solar neutrino data from the Sudbury Neutrino Observatory, Phys. Rev. C 88 (2013) 025501 [arXiv: 1109.0763] [INSPIRE].

[13] G. Bellini et al., Precision measurement of the ${ }^{7}$ Be solar neutrino interaction rate in Borexino, Phys. Rev. Lett. 107 (2011) 141302 [arXiv:1104.1816] [INSPIRE].

[14] Borexino collaboration, M. Agostini et al., First simultaneous precision spectroscopy of pp, ${ }^{7}$ Be and pep solar neutrinos with Borexino Phase-II, arXiv:1707.09279 [InSPIRE].

[15] Super-Kamiokande collaboration, K. Abe et al., Atmospheric neutrino oscillation analysis with external constraints in Super-Kamiokande I-IV, Phys. Rev. D 97 (2018) 072001 [arXiv: 1710.09126] [INSPIRE].

[16] IceCube collaboration, M.G. Aartsen et al., Measurement of Atmospheric Neutrino Oscillations at 6-56 GeV with IceCube DeepCore, Phys. Rev. Lett. 120 (2018) 071801 [arXiv: 1707.07081] [INSPIRE].

[17] KamLAND collaboration, A. Gando et al., Reactor on-off antineutrino measurement with KamLAND, Phys. Rev. D 88 (2013) 033001 [arXiv:1303.4667] [INSPIRE].

[18] DaYa Bay collaboration, F.P. An et al., Measurement of electron antineutrino oscillation based on 1230 days of operation of the Daya Bay experiment, Phys. Rev. D 95 (2017) 072006 [arXiv : 1610.04802] [INSPIRE].

[19] Double CHOOZ collaboration, Y. Abe et al., Improved measurements of the neutrino mixing angle $\theta_{13}$ with the Double CHOOZ detector, JHEP 10 (2014) 086 [Erratum ibid. 02 (2015) 074] [arXiv: 1406.7763] [INSPIRE].

[20] A. Minotti, Latest results from Double CHOOZ, Phys. Part. Nucl. 48 (2017) 47 [InSPIRE]. 
[21] RENO collaboration, J.K. Ahn et al., Observation of reactor electron antineutrino disappearance in the RENO experiment, Phys. Rev. Lett. 108 (2012) 191802 [arXiv:1204.0626] [INSPIRE].

[22] RENO collaboration, M.Y. Pac, Recent results from RENO, PoS (NuFact2017) 038 [arXiv: 1801.04049] [INSPIRE].

[23] K2K collaboration, M.H. Ahn et al., Measurement of neutrino oscillation by the K2K experiment, Phys. Rev. D 74 (2006) 072003 [hep-ex/0606032] [INSPIRE].

[24] MINOS collaboration, P. Adamson et al., Electron neutrino and antineutrino appearance in the full MINOS data sample, Phys. Rev. Lett. 110 (2013) 171801 [arXiv:1301.4581] [INSPIRE].

[25] MINOS collaboration, P. Adamson et al., Measurement of neutrino and antineutrino oscillations using beam and atmospheric data in MINOS, Phys. Rev. Lett. 110 (2013) 251801 [arXiv: 1304.6335] [INSPIRE].

[26] T2K collaboration, K. Abe et al., Combined analysis of neutrino and antineutrino oscillations at T2K, Phys. Rev. Lett. 118 (2017) 151801 [arXiv:1701.00432] [INSPIRE].

[27] T2K collaboration, K. Abe et al., Updated T2K measurements of muon neutrino and antineutrino disappearance using $1.5 \times 10^{21}$ protons on target, Phys. Rev. D 96 (2017) 011102 [arXiv: 1704.06409] [INSPIRE].

[28] NOvA collaboration, P. Adamson et al., Measurement of the neutrino mixing angle $\theta_{23}$ in NOvA, Phys. Rev. Lett. 118 (2017) 151802 [arXiv:1701.05891] [INSPIRE].

[29] NOvA collaboration, P. Adamson et al., Constraints on oscillation parameters from $\nu_{e}$ appearance and $\nu_{\mu}$ disappearance in NOvA, Phys. Rev. Lett. 118 (2017) 231801 [arXiv: 1703.03328] [INSPIRE].

[30] I. Esteban et al., Updated fit to three neutrino mixing: exploring the accelerator-reactor complementarity, JHEP 01 (2017) 087 [arXiv:1611.01514] [INSPIRE].

[31] F. Capozzi et al., Global constraints on absolute neutrino masses and their ordering, Phys. Rev. D 95 (2017) 096014 [arXiv:1703.04471] [INSPIRE].

[32] P.F. de Salas et al., Status of neutrino oscillations 2018: $3 \sigma$ hint for normal mass ordering and improved CP sensitivity, Phys. Lett. B 782 (2018) 633 [arXiv:1708.01186] [INSPIRE].

[33] K.N. Abazajian et al., Light sterile neutrinos: a white paper, arXiv:1204.5379 [INSPIRE].

[34] G.H. Collin, C.A. Argüelles, J.M. Conrad and M.H. Shaevitz, First constraints on the complete neutrino mixing matrix with a sterile neutrino, Phys. Rev. Lett. 117 (2016) 221801 [arXiv:1607.00011] [INSPIRE].

[35] S. Gariazzo, C. Giunti, M. Laveder and Y.F. Li, Updated global $3+1$ analysis of short-baseline neutrino oscillations, JHEP 06 (2017) 135 [arXiv:1703.00860] [INSPIRE].

[36] LSND collaboration, C. Athanassopoulos et al., Candidate events in a search for anti-muon-neutrino to anti-electron-neutrino oscillations, Phys. Rev. Lett. 75 (1995) 2650 [nucl-ex/9504002] [INSPIRE].

[37] LSND collaboration, A. Aguilar-Arevalo et al., Evidence for neutrino oscillations from the observation of anti-neutrino(electron) appearance in a anti-neutrino(muon) beam, Phys. Rev. D 64 (2001) 112007 [hep-ex/0104049] [INSPIRE].

[38] G. Mention et al., The reactor antineutrino anomaly, Phys. Rev. D 83 (2011) 073006 [arXiv:1101.2755] [INSPIRE]. 
[39] T.A. Mueller et al., Improved predictions of reactor antineutrino spectra, Phys. Rev. C 83 (2011) 054615 [arXiv: 1101.2663] [INSPIRE].

[40] P. Huber, On the determination of anti-neutrino spectra from nuclear reactors, Phys. Rev. C 84 (2011) 024617 [Erratum ibid. C 85 (2012) 029901] [arXiv:1106.0687] [INSPIRE].

[41] NEOS collaboration, Y. Ko et al., Sterile neutrino search at the NEOS experiment, Phys. Rev. Lett. 118 (2017) 121802 [arXiv: 1610.05134] [INSPIRE].

[42] M. Danilov, Search for sterile neutrinos at the DANSS experiment, talk given at the DANSS experiment at the Solvay Workshop: Beyond the Standard model with Neutrinos and Nuclear Physics, November 29-December 1, Brussels, Belgium (2017).

[43] S. Gariazzo, C. Giunti, M. Laveder and Y.F. Li, Model-independent $\bar{\nu}_{e}$ short-baseline oscillations from reactor spectral ratios, Phys. Lett. B 782 (2018) 13 [arXiv:1801.06467] [INSPIRE].

[44] I.E. Stockdale et al., Limits on muon neutrino oscillations in the mass range $55 \mathrm{eV}^{2}<\Delta m^{2}<800 e V^{2}$, Phys. Rev. Lett. 52 (1984) 1384 [INSPIRE].

[45] F. Dydak et al., A search for muon-neutrino oscillations in the $\Delta m^{2}$ Range $0.3 \mathrm{eV}^{2}$ to $90 \mathrm{eV}^{2}$, Phys. Lett. B 134 (1984) 281.

[46] SciBoone, MiniBoone collaboration, K.B.M. Mahn et al., Dual baseline search for muon neutrino disappearance at $0.5 \mathrm{eV}^{2}<\Delta m^{2}<40 \mathrm{eV}^{2}$, Phys. Rev. D 85 (2012) 032007 [arXiv:1106.5685] [INSPIRE].

[47] SciBoone, MiniBoone collaboration, G. Cheng et al., Dual baseline search for muon antineutrino disappearance at $0.1 \mathrm{eV}^{2}<\Delta m^{2}<100 \mathrm{eV}^{2}$, Phys. Rev. D 86 (2012) 052009 [arXiv: 1208.0322] [INSPIRE].

[48] MINOS collaboration, P. Adamson et al., Search for sterile neutrinos mixing with muon neutrinos in MINOS, Phys. Rev. Lett. 117 (2016) 151803 [arXiv:1607.01176] [INSPIRE].

[49] Super-Kamiokande collaboration, K. Abe et al., Limits on sterile neutrino mixing using atmospheric neutrinos in Super-Kamiokande, Phys. Rev. D 91 (2015) 052019

[arXiv: 1410.2008] [INSPIRE].

[50] ICECube collaboration, M.G. Aartsen et al., Searches for sterile neutrinos with the IceCube detector, Phys. Rev. Lett. 117 (2016) 071801 [arXiv: 1605.01990] [InSPIRE].

[51] F. Capozzi, C. Giunti, M. Laveder and A. Palazzo, Joint short- and long-baseline constraints on light sterile neutrinos, Phys. Rev. D 95 (2017) 033006 [arXiv:1612.07764] [INSPIRE].

[52] M. Dentler et al., Sterile neutrinos or flux uncertainties? - Status of the reactor anti-neutrino anomaly, JHEP 11 (2017) 099 [arXiv: 1709.04294] [INSPIRE].

[53] M. Dentler et al., Updated global analysis of neutrino oscillations in the presence of eV-scale sterile neutrinos, arXiv:1803.10661 [INSPIRE].

[54] B.K. Cogswell et al., Neutrino oscillations: ILL experiment revisited, arXiv:1802.07763 [INSPIRE].

[55] Sld Electroweak Group, DelPhi, AlePh, SLD, SLD Heavy Flavour Group, OPAL, LEP Electroweak Working Group, L3 collaboration, S. Schael et al., Precision electroweak measurements on the $Z$ resonance, Phys. Rept. 427 (2006) 257 [hep-ex/0509008] [INSPIRE].

[56] S. Gariazzo, C. Giunti, M. Laveder, Y.F. Li and E.M. Zavanin, Light sterile neutrinos, J. Phys. G 43 (2016) 033001 [arXiv: 1507.08204] [INSPIRE]. 
[57] C. Giunti, Light Sterile Neutrinos: Status and Perspectives, Nucl. Phys. B 908 (2016) 336 [arXiv: 1512.04758] [INSPIRE].

[58] L. Stanco, Search for sterile neutrinos at long and short baselines, in the proceedings of Prospects in Neutrino Physics (NuPhys2015), December 16-18, London, U.K. (2015), arXiv: 1604.06769 [INSPIRE].

[59] A. Fava, Experimental investigation of the thriving mystery of sterile neutrinos, Rev. Phys. 1 (2016) 52.

[60] N. Klop and A. Palazzo, Imprints of CP-violation induced by sterile neutrinos in T2K data, Phys. Rev. D 91 (2015) 073017 [arXiv:1412.7524] [INSPIRE].

[61] S.K. Agarwalla et al., Discovery potential of T2K and NOvA in the presence of a light sterile neutrino, JHEP 02 (2016) 111 [arXiv:1601.05995] [INSPIRE].

[62] M. Ghosh, S. Gupta, Z.M. Matthews, P. Sharma and A.G. Williams, Study of parameter

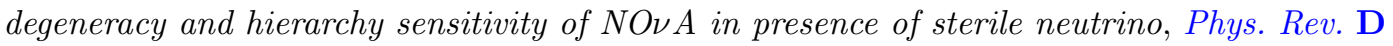
96 (2017) 075018 [arXiv: 1704.04771] [INSPIRE].

[63] R. Gandhi, B. Kayser, M. Masud and S. Prakash, The impact of sterile neutrinos on CP measurements at long baselines, JHEP 11 (2015) 039 [arXiv:1508.06275] [INSPIRE].

[64] S.K. Agarwalla, S.S. Chatterjee and A. Palazzo, Octant of $\theta_{23}$ in danger with a light sterile neutrino, Phys. Rev. Lett. 118 (2017) 031804 [arXiv: 1605. 04299] [INSPIRE].

[65] S.K. Agarwalla, S.S. Chatterjee and A. Palazzo, Degeneracy between $\theta_{23}$ octant and neutrino non-standard interactions at DUNE, Phys. Lett. B 762 (2016) 64 [arXiv: 1607.01745] [INSPIRE].

[66] S. Choubey, D. Dutta and D. Pramanik, Imprints of a light sterile neutrino at DUNE, T2HK and T2HKK, Phys. Rev. D 96 (2017) 056026 [arXiv:1704.07269] [InSPIRE].

[67] S.K. Agarwalla, S.S. Chatterjee and A. Palazzo, Signatures of a light sterile neutrino in T2HK, JHEP 04 (2018) 091 [arXiv: 1801.04855] [INSPIRE].

[68] S. Razzaque and A.Yu. Smirnov, Searching for sterile neutrinos in ice, JHEP 07 (2011) 084 [arXiv:1104.1390] [INSPIRE].

[69] A. Esmaili, F. Halzen and O.L.G. Peres, Constraining sterile neutrinos with AMANDA and IceCube atmospheric neutrino data, JCAP 11 (2012) 041 [arXiv:1206.6903] [INSPIRE].

[70] A. Esmaili and A.Yu. Smirnov, Restricting the LSND and MiniBooNE sterile neutrinos with the IceCube atmospheric neutrino data, JHEP 12 (2013) 014 [arXiv:1307.6824] [INSPIRE].

[71] M. Blennow et al., IceCube bounds on sterile neutrinos above $10 \mathrm{eV}$, arXiv:1803.02362 [INSPIRE].

[72] S. Razzaque and A.Yu. Smirnov, Searches for sterile neutrinos with IceCube DeepCore, Phys. Rev. D 85 (2012) 093010 [arXiv:1203.5406] [INSPIRE].

[73] A. Esmaili, F. Halzen and O.L.G. Peres, Exploring $\nu_{\tau}-\nu_{s}$ mixing with cascade events in DeepCore, JCAP 07 (2013) 048 [arXiv:1303.3294] [INSPIRE].

[74] S. Choubey, N.P. Harries and G.G. Ross, Turbulent supernova shock waves and the sterile neutrino signature in megaton water detectors, Phys. Rev. D 76 (2007) 073013 [hep-ph/0703092] [INSPIRE].

[75] A. Esmaili, O.L.G. Peres and P.D. Serpico, Impact of sterile neutrinos on the early time flux from a galactic supernova, Phys. Rev. D 90 (2014) 033013 [arXiv:1402.1453] [INSPIRE]. 
[76] M. Archidiacono, E. Giusarma, S. Hannestad and O. Mena, Cosmic dark radiation and neutrinos, Adv. High Energy Phys. 2013 (2013) 191047 [arXiv: 1307.0637] [INSPIRE].

[77] J. Lesgourgues and S. Pastor, Neutrino cosmology and Planck, New J. Phys. 16 (2014) 065002 [arXiv: 1404.1740] [INSPIRE].

[78] P.C. de Holanda and A.Yu. Smirnov, Homestake result, sterile neutrinos and low-energy solar neutrino experiments, Phys. Rev. D 69 (2004) 113002 [hep-ph/0307266] [InSPIRE].

[79] P.C. de Holanda and A.Yu. Smirnov, Solar neutrino spectrum, sterile neutrinos and additional radiation in the Universe, Phys. Rev. D 83 (2011) 113011 [arXiv:1012.5627] [INSPIRE].

[80] P.S. Bhupal Dev and A. Pilaftsis, Light and superlight sterile neutrinos in the minimal radiative inverse seesaw model, Phys. Rev. D 87 (2013) 053007 [arXiv:1212.3808] [INSPIRE].

[81] W. Liao, Y. Luo and X.-H. Wu, Effect of interaction with neutrons in matter on flavor conversion of super-light sterile neutrino with active neutrino, JHEP 06 (2014) 069 [arXiv: 1403.2559] [INSPIRE].

[82] P.C. Divari and J.D. Vergados, Neutrino oscillations in the presence of super-light sterile neutrinos, Int. J. Mod. Phys. A 31 (2016) 1650123 [arXiv: 1602.08690] [INSPIRE].

[83] A. Palazzo, Constraints on very light sterile neutrinos from $\theta_{13}$-sensitive reactor experiments, JHEP 10 (2013) 172 [arXiv:1308.5880] [INSPIRE].

[84] A. Esmaili et al., Probing light sterile neutrinos in medium baseline reactor experiments, Phys. Rev. D 88 (2013) 073012 [arXiv:1308.6218] [INSPIRE].

[85] I. Girardi et al., Constraining sterile neutrinos using reactor neutrino experiments, JHEP 08 (2014) 057 [arXiv: 1405.6540] [INSPIRE].

[86] Daya Bay collaboration, F.P. An et al., Search for a light sterile neutrino at Daya Bay, Phys. Rev. Lett. 113 (2014) 141802 [arXiv:1407.7259] [INSPIRE].

[87] DaYa BAY collaboration, F.P. An et al., Improved search for a light sterile neutrino with the full configuration of the Daya Bay experiment, Phys. Rev. Lett. 117 (2016) 151802 [arXiv: 1607.01174] [INSPIRE].

[88] D.O. Caldwell, G.M. Fuller and Y.-Z. Qian, Sterile neutrinos and supernova nucleosynthesis, Phys. Rev. D 61 (2000) 123005 [astro-ph/9910175] [INSPIRE].

[89] I. Tamborra, G.G. Raffelt, L. Hudepohl and H.-T. Janka, Impact of eV-mass sterile neutrinos on neutrino-driven supernova outflows, JCAP 01 (2012) 013 [arXiv:1110.2104] [INSPIRE].

[90] M.-R. Wu et al., Impact of active-sterile neutrino mixing on supernova explosion and nucleosynthesis, Phys. Rev. D 89 (2014) 061303 [arXiv:1305.2382] [INSPIRE].

[91] T. Asaka, S. Blanchet and M. Shaposhnikov, The $\nu M S M$, dark matter and neutrino masses, Phys. Lett. B 631 (2005) 151 [hep-ph/0503065] [InSPIRE].

[92] T. Asaka and M. Shaposhnikov, The $\nu M S M$, dark matter and baryon asymmetry of the universe, Phys. Lett. B 620 (2005) 17 [hep-ph/0505013] [InSPIRE].

[93] A. Boyarsky, O. Ruchayskiy and M. Shaposhnikov, The role of sterile neutrinos in cosmology and astrophysics, Ann. Rev. Nucl. Part. Sci. 59 (2009) 191 [arXiv:0901.0011] [INSPIRE]. 
[94] M. Drewes et al., A white paper on keV sterile neutrino dark matter, JCAP 01 (2017) 025 [arXiv: 1602.04816] [INSPIRE].

[95] ICAL collaboration, S. Ahmed et al., Physics potential of the ICAL detector at the India-based Neutrino Observatory (INO), Pramana 88 (2017) 79 [arXiv:1505.07380] [INSPIRE].

[96] India-based Neutrino Observatory (INO), http://www.ino.tifr.res.in/ino/.

[97] A. Chatterjee et al., A simulations study of the muon response of the iron calorimeter detector at the India-based Neutrino Observatory, 2014 JINST 9 P07001 [arXiv: 1405.7243] [INSPIRE].

[98] M.M. Devi, A. Ghosh, D. Kaur, L.S. Mohan, S. Choubey, A. Dighe et al., Hadron energy response of the Iron Calorimeter detector at the India-based Neutrino Observatory, 2013 JINST 8 P11003 [arXiv:1304.5115] [INSPIRE].

[99] L.S. Mohan et al., Simulation studies of hadron energy resolution as a function of iron plate thickness at INO-ICAL, 2014 JINST 9 T09003 [arXiv:1401.2779] [INSPIRE].

[100] A. Ghosh, T. Thakore and S. Choubey, Determining the neutrino mass hierarchy with INO, T2K, NOvA and reactor experiments, JHEP 04 (2013) 009 [arXiv: 1212.1305] [INSPIRE].

[101] M.M. Devi et al., Enhancing sensitivity to neutrino parameters at INO combining muon and hadron information, JHEP 10 (2014) 189 [arXiv:1406.3689] [INSPIRE].

[102] A. Ajmi et al., Improving the hierarchy sensitivity of ICAL using neural network, J. Phys. Conf. Ser. 888 (2017) 012151 [arXiv:1510.02350] [INSPIRE].

[103] T. Thakore et al., The reach of INO for atmospheric neutrino oscillation parameters, JHEP 05 (2013) 058 [arXiv: 1303.2534] [INSPIRE].

[104] D. Kaur, M. Naimuddin and S. Kumar, The sensitivity of the ICAL detector at India-based Neutrino Observatory to neutrino oscillation parameters, Eur. Phys. J. C 75 (2015) 156 [arXiv: 1409.2231] [INSPIRE].

[105] L.S. Mohan and D. Indumathi, Pinning down neutrino oscillation parameters in the 2 sector with a magnetised atmospheric neutrino detector: a new study, Eur. Phys. J. C 77 (2017) 54 [arXiv: 1605.04185] [INSPIRE].

[106] S.P. Behera et al., Search for the sterile neutrino mixing with the ICAL detector at INO, Eur. Phys. J. C 77 (2017) 307 [arXiv: 1605.08607] [InSPIRE].

[107] S. Goswami, Accelerator, reactor, solar and atmospheric neutrino oscillation: Beyond three generations, Phys. Rev. D 55 (1997) 2931 [hep-ph/9507212] [INSPIRE].

[108] N. Okada and O. Yasuda, A Sterile neutrino scenario constrained by experiments and cosmology, Int. J. Mod. Phys. A 12 (1997) 3669 [hep-ph/9606411] [INSPIRE].

[109] S.M. Bilenky, C. Giunti and W. Grimus, Neutrino mass spectrum from the results of neutrino oscillation experiments, Eur. Phys. J. C 1 (1998) 247 [hep-ph/9607372] [INSPIRE].

[110] S.M. Bilenky et al., Four neutrino mass spectra and the Super-Kamiokande atmospheric up-down asymmetry, Phys. Rev. D 60 (1999) 073007 [hep-ph/9903454] [INSPIRE].

[111] M. Maltoni, T. Schwetz, M.A. Tortola and J.W.F. Valle, Status of global fits to neutrino oscillations, New J. Phys. 6 (2004) 122 [hep-ph/0405172] [INSPIRE].

[112] Planck collaboration, P.A.R. Ade et al., Planck 2015 results. XIII. Cosmological parameters, Astron. Astrophys. 594 (2016) A13 [arXiv:1502.01589] [INSPIRE]. 
[113] MINOS, Daya Bay collaboration, P. Adamson et al., Limits on active to sterile neutrino oscillations from disappearance searches in the MINOS, Daya Bay and Bugey-3 experiments, Phys. Rev. Lett. 117 (2016) 151801 [arXiv:1607.01177] [INSPIRE].

[114] IceCube collaboration, M.G. Aartsen et al., Search for sterile neutrino mixing using three years of IceCube DeepCore data, Phys. Rev. D 95 (2017) 112002 [arXiv:1702.05160] [INSPIRE].

[115] MINOS collaboration, P. Adamson et al., Active to sterile neutrino mixing limits from neutral-current interactions in MINOS, Phys. Rev. Lett. 107 (2011) 011802 [arXiv: 1104.3922] [INSPIRE].

[116] NOvA collaboration, P. Adamson et al., Search for active-sterile neutrino mixing using neutral-current interactions in NOvA, Phys. Rev. D 96 (2017) 072006 [arXiv:1706. 04592] [INSPIRE].

[117] V.D. Barger et al., Neutrino mixing, CP/T violation and textures in four neutrino models, Phys. Rev. D 59 (1999) 113010 [hep-ph/9901388] [INSPIRE].

[118] B. Kayser, Neutrino mass, mixing and flavor change, hep-ph/0211134 [INSPIRE].

[119] P. Huber, M. Lindner and W. Winter, Simulation of long-baseline neutrino oscillation experiments with GLoBES (General Long Baseline Experiment Simulator), Comput. Phys. Commun. 167 (2005) 195 [hep-ph/0407333] [INSPIRE].

[120] P. Huber et al., New features in the simulation of neutrino oscillation experiments with GLoBES 3.0: General Long Baseline Experiment Simulator, Comput. Phys. Commun. 177 (2007) 432 [hep-ph/0701187] [INSPIRE].

[121] A.M. Dziewonski and D. L. Anderson, Preliminary reference Earth model, Phys. Earth Planet. Inter. 25 (1981) 297.

[122] D. Casper, The Nuance neutrino physics simulation and the future, Nucl. Phys. Proc. Suppl. 112 (2002) 161 [hep-ph/0208030] [INSPIRE].

[123] P. Huber, M. Lindner and W. Winter, Superbeams versus neutrino factories, Nucl. Phys. B 645 (2002) 3 [hep-ph/0204352] [INSPIRE].

[124] G.L. Fogli et al., Solar neutrino oscillation parameters after first KamLAND results, Phys. Rev. D 67 (2003) 073002 [hep-ph/0212127] [INSPIRE].

[125] M.C. Gonzalez-Garcia and M. Maltoni, Atmospheric neutrino oscillations and new physics, Phys. Rev. D 70 (2004) 033010 [hep-ph/0404085] [INSPIRE].

[126] E.K. Akhmedov et al., Series expansions for three flavor neutrino oscillation probabilities in matter, JHEP 04 (2004) 078 [hep-ph/0402175] [INSPIRE].

[127] MINOS collaboration, P. Adamson et al., Search for sterile neutrinos in MINOS and MINOS+ using a two-detector fit, submitted to Phys. Rev. Lett. (2017), [arXiv:1710.06488 [INSPIRE]. 